\title{
Generalisations of coarse spaces *
}

\author{
Nicolò Zava ${ }^{\dagger}$ \\ Department of Mathematical, Computer and Physical Sciences, University of Udine \\ Via Delle Scienze 206, 33100 Udine, Italy \\ nicolo.zava@gmail.com
}

\begin{abstract}
Coarse geometry, the branch of topology that studies the global properties of spaces, was originally developed for metric spaces and then Roe introduced coarse structures ([21]) as a large-scale counterpart of uniformities. In the literature, there are very important generalisations of uniform spaces, such as semi-uniform and quasi-uniform spaces. In this paper, we introduce and start to study their large-scale counterparts, which generalise coarse spaces: semi-coarse spaces and quasi-coarse spaces.
\end{abstract}

\section{Introduction}

Large-scale geometry, or coarse geometry, is the study of the global properties of spaces, ignoring their local, smallscale ones. It was initially developed for metric spaces and it found many important applications in different branches of mathematics (see [13] for an overview). For example, here we mention the applications to geometric group theory, where a finitely generated group has essentially two structures of metric space induced by its word metrics, up to (see [8]). Roe introduced coarse spaces ([21]) in order to encode the large-scale properties of spaces and to extend that approach outside the realm of metric spaces. His definition is very similar to the one of uniform space. Recall that a uniform space is a pair $(X, \mathcal{U})$, where $X$ is a set and $\mathcal{U}$ is a uniformity over it, i.e., a family of subsets of $X \times X$, called entourages, that satisfy the following properties:

(U1) $\mathcal{U}$ is a filter (i.e., a family closed under taking finite intersections and supersets);

'(U2) for every $U \in \mathcal{U}, \Delta_{X}=\{(x, x) \mid x \in X\} \subseteq U$

(U3) for every $\left.U \in \mathcal{U}, U^{-1}=\{(x, y) \mid(y, x) \in \bar{U}\} \in \mathcal{U}\right\}$;

'(U4) for every $U \in \mathcal{U}$, there exists $V \in \mathcal{U}$ such that $V \circ V \subseteq U$ (for every pair of entourages $E, F \subseteq X \times X$, denote $E \circ F=\{(x, z) \mid \exists y \in X:(x, y) \in E,(y, z) \in F\})$.

For instance, if $(X, d)$ is a metric space, then, if we denote by $B_{d}(x, R)$ the open ball centered in $x \in X$ with radius $R>0$, the family

$$
\mathcal{U}_{d}=\left\{V \subseteq U_{R} \mid R \geq 0\right\}, \text { where, for every } R>0, U_{R}=\bigcup_{x \in X}\{x\} \times B_{d}(x, R),
$$

is a uniformity over $X$. Dydak and Hoffland in [7] and Protasov in [16] independently introduced the large-scale counterparts of the approach to uniformities via coverings. Let us also cite balleans, which are structures equivalent to coarse spaces, that Protasov and Banakh defined to generalise metric balls ([17]).

Uniform spaces have been widely studied since their introduction by the work of Weil and Tukey in the first half of the last century, and successfully applied in different areas. We refer to [10] for an introduction to the topic. However, in some cases, uniform spaces have too strong axioms and they cannot parametrise important situations. For example a quasi-metric of a set $X$ is a map $d: X \times X \rightarrow \mathbb{R}_{\geq 0}$ that satisfies all the axioms of a metric but the symmetry. Quasimetrics naturally arises in many situations, for instance they were cited already in Hausdorff monograph [9], when he discussed the Hausdorff metric of a metric space. Moreover, if we allow that $d$ may also take the value $\infty$, then preorders can be described as quasi-metrics. We refer to [22] for a general introduction to the subject. Quasi-metrics are innerlu non symmetric, so, if we consider the family $\mathscr{U}_{d}$ as in (11), then (U3) may not be satisfied.

In order to fill the gap, quasi-uniform spaces were introduced: a quasi-uniform space is a pair $(X, \mathcal{U})$, where $\mathcal{U}$ is a quasi-uniformity over the set $X$, i.e. a family of entourages that satisfies (U1), (U2) and (U4). There is a wide literature investigating those structures and also important applications to computer science were discovered (see the monograph [11] and the survey [12 for a wide-range introduction and a broad bibliography). Similarly, a semi-uniform space is a pair $(X, \mathcal{U})$, where $\mathcal{U}$ is a semi-uniformity over the set $X$, i.e., a family of entourages that satisfies (U1)-(U3) (see, for example, [3]).

\footnotetext{
*MSC: 18B99, 54E15, 54E25, 54E99, 54A99.

Keywords: coarse space, quasi-coarse space, semi-coarse space, bornologous map, monoid, coarse equivalence, Sym-coarse equivalence.

†This work was partially supported by the "National Group of Algebraic and Geometric Structures, and their Applications" (GNSAGA INdAM).
} 
The aim of this paper is to introduce large-scale counterparts of quasi-uniform spaces and semi-uniform spaces, respectively, in order to generalise coarse spaces. In particular, we define quasi-coarse spaces and semi-coarse spaces (Definition 1.1). Moreover, in order to provide a more comprehensive introduction to these new objects, we consider also entourage spaces, which are structures that generalise both quasi-coarse spaces and semi-coarse spaces. First of all, scratching the surface of this topic, we focus on adapting basic notions of coarse geometry (e.g., morphisms, as bornologous maps, connectedness, boundedness) to this more general setting. Moreover, we present a different characterisation of those structures by using ball structures ([17]).

We motivate our interest in quasi-coarse spaces and semi-coarse spaces by providing a wide list of examples in which those structures naturally appear. Most of them are extensions of some classical examples of coarse spaces. For instance, we widely discuss metric entourage structures (e.g., the structures induced by quasi-metrics), relation entourage structures (for example the ones induced by a preorder on a set), graphic quasi-coarse structures on directed graphs, hyperstructures, finitely generated monoids endowed with word quasi-metrics, and entourage structures on unitary magmas, monoids, and loops. In particular, we prove that every finitely generated monoid can be endowed with precisely just two word quasi-metrics up to asymorphism (Proposition 2.3), which coincide if the monoid is abelian. This result is a generalisation of the classical situation with finitely generated groups endowed with word metrics.

In studying these structures, it is useful to pursue a categorical approach to the subject. We then introduce the categories Entou, QCoarse, and SCoarse of entourage spaces, quasi-coarse spaces, and semi-coarse spaces, respectively, and bornologous maps between them. The category Coarse, of coarse spaces and bornologous maps between them, which was previously studied in [6], is a full subcategory of all those categories. In the same paper, it was shown that Coarse is topological and we extend this result to Entou, QCoarse, and SCoarse (Theorem 3.2).

The existence of several functors between those four categories turned out to be very useful in a twofold way. First of all, we use them to prove that QCoarse is a reflective subcategory in Entou (but it is not co-reflective), SCoarse is a reflective and co-reflective subcategory in Entou, Coarse is a reflective subcategory in SCoarse (but it is not coreflective), and Coarse is a reflective and co-reflective subcategory in QCoarse (see Theorem 3.6 and \$3.2). This result helps us in defining some basic categorical constructions, such as products, coproducts and quotients. Furthermore, those functors are a fundamental tool to transport the important notion of closeness of morphisms (and thus coarse equivalences, see [21]) from coarse spaces, to the other, weaker structures. In particular, we focus on the notion of Sym-coarse equivalence between quasi-coarse spaces and we provide a characterisation of that property that is similar to the classical one for coarse equivalences between coarse spaces (see Theorem 4.4).

Finally, we use Sym-coarse equivalences to give important characterisations of some classes of quasi-coarse spaces: metric entourage spaces induced by extended-quasi-metrics and graphic quasi-coarse spaces, giving an answer to a problem posed by Protasov and Banakh ([17, Problem 9.4]).

The paper is organised as follows. In Section 1 we recall some background in coarse geometry, while we introduce some new notions. For example, here the definitions of quasi-coarse space, semi-coarse space, entourage space are given. Moreover, one of the most important example of those structures, metric entourage structures, is provided and the entourage structures or semi-coarse structures induced by extended semi-positive-definite maps and by extended semipseudometric, respectively, are characterised. Moreover, 1.1 is devoted to discuss morphisms between those spaces and in $₫ 1.2$ an equivalent description of those structures by using ball structures is presented. Providing examples of entourage structures is the focus of Section 2, We widely discuss relation entourage structures (\$2.1), graphic quasi-coarse structures $(\$ 2.2)$, hyperstructures $(\$ 2.3)$, finitely generated monoids $(2.4)$ and other entourage structures on algebraic structures, such as unitary magmas, monoids, and loops (2.5). In Section 3 the categorical approach is presented. We define the four categories Entou, SCoarse, QCoarse, and Coarse and we prove that they are topological. Moreover, we provide functors between them and show that QCoarse is a reflective subcategory in Entou, SCoarse is a reflective and co-reflective subcategory in Entou, Coarse is a reflective subcategory in SCoarse, and Coarse is a reflective and co-reflective subcategory in QCoarse (3.1). We conclude the section by presenting some categorical constructions, such as products, coproducts and quotients (\$3.2). Finally, in Section 4, we introduce the notion of F-coarse equivalence, where $\mathrm{F}$ is a functor from a category $\mathcal{X}$ to Coarse. In particular, we focus on the functor Sym: QCoarse $\rightarrow$ Coarse and we characterise Sym-coarse equivalences. We conclude the paper by characterising some special classes of quasi-coarse spaces (4.1).

\section{Generalisations of coarse spaces}

Let $X$ be a set. An entourage is a subset of the product $X \times X$. For every entourage $E$, every point $x \in X$, and every subset $A$ of $X$, denote

$$
E[x]=\{y \in X \mid(x, y) \in E\}, \quad E[A]=\bigcup_{a \in A} E[a] .
$$

Let $X$ be a set. An ideal on $X$ is a family $\mathcal{I}$ of subsets of $X$ which is closed under taking subsets and finite unions. Moreover, if $\mathcal{F}$ is a family of subsets of $X$, we denote by $\widehat{\mathcal{F}}$ its closure under taking subsets, i.e., $\widehat{\mathcal{F}}=\{A \subseteq F \mid F \in \mathcal{F}\}$.

Definition 1.1. Let $X$ be a set. A family $\mathcal{E} \subseteq \mathcal{P}(X \times X)$ is an entourages structure over $X$ if it is an ideal on $X \times X$ that contains the diagonal $\Delta_{X}$. Moreover, an entourages structure $\mathcal{E}$ over $X$ is

- a semi-coarse structure if $E^{-1} \in \mathcal{E}$, for every $E \in \mathcal{E}$; 
- a quasi-coarse structure if $E \circ F \in \mathcal{E}$, for every $E, F \in \mathcal{E}$;

- a coarse structure if it is both a semi-coarse and a quasi-coarse structure.

The pair $(X, \mathcal{E})$ is an entourages space (a semi-coarse space, a quasi-coarse space, a coarse space) if $\mathcal{E}$ is an entourages structure (a semi-coarse structure, a quasi-coarse structure, a coarse structure, respectively) over $X$.

If $\mathcal{E}$ is an entourage structure on a set $X$, then also $\mathcal{E}^{-1}=\left\{E^{-1} \mid E \in \mathcal{E}\right\}$ is an entourage structure. Of course, $\mathcal{E}=\mathcal{E}^{-1}$ if and only if $\mathcal{E}$ is a semi-structure. Moreover, if $\mathcal{E}$ is a quasi-coarse structure, then $\mathcal{E}^{-1}$ is a quasi-coarse structure.

Let $(X, \mathcal{E})$ be an entourage space and $Y$ be a subset of $X$. Then $Y$ can be endowed with the entourage substructure $\left.\mathcal{E}\right|_{Y}=\{E \cap(Y \times Y) \mid E \in \mathcal{E}\}$, and $\left(Y,\left.\mathcal{E}\right|_{Y}\right)$ is called an entourage subspace of $(X, \mathcal{E})$. If $\mathcal{E}$ is a quasi-coarse structure (semi-coarse structure), then $\left.\mathcal{E}\right|_{Y}$ is a quasi-coarse structure (semi-coarse structure, respectively).

If $X$ is a set, a family $\mathcal{B}$ of subsets of $X \times X$ such that $\mathcal{E}=\widehat{\mathcal{B}}$ is an entourages structure (semi-coarse structure, quasi-coarse structure, coarse structure, respectively) is a base for the entourages structure (base for the semi-coarse structure, base for the quasi-coarse structure, base for the coarse structure, respectively) $\mathcal{E}$.

Let us now give the most important example of these structures.

Example 1.2. Let $X$ be a set and $d: X \times X \rightarrow[0, \infty)$ be a map such that $d(x, x)=0$, for every $x \in X$. The map $d$ is a semi-positive-definite map. Moreover $d$ is a

- semi-pseudometric if, for every $x, y \in X, d(x, y)=d(y, x)$;

- quasi-pseudometric if, for every $x, y, z \in X, d(x, y) \leq d(x, z)+d(z, y)$;

- pseudometric if it is both a quasi-pseudometric and a semi-pseudometric.

Semi-pseudometrics, quasi-pseudometrics and metrics are semi-metrics, quasi-metrics and metrics, respectively, if $x=y$ whenever $d(x, y)=0$. If $d: X \times X \rightarrow[0,+\infty]$, then the function is called extended. Moreover the pair $(X, d)$ is called a (extended) semi-pseudometric space, a (extended) quasi-pseudometric space, a (extended) pseudometric space, a (extended) semi-metric space, a (extended) quasi-metric space, a (extended) metric space, respectively.

A leading example of entourage structures is the metric entourage structure. Let $(X, d)$ be a set endowed with an extended semi-positive-definite map $d$. We define the following entourage structure:

$$
\mathcal{E}_{d}=\left\{E_{R} \subseteq \widehat{X \times X} \mid R \geq 0\right\}, \quad \text { where, for every } R \geq 0, \quad E_{R}=\bigcup_{x \in X}\left(\{x\} \times B_{d}(x, R)\right) .
$$

Even though it is not precise, for the sake of simplicity, we call $\mathcal{E}_{d}$ a metric entourage structure. If $d$ is an extended semipseudometric, then $\mathcal{E}_{d}$ is a semi-coarse structure, while, if $d$ is an extended quasi-pseudometric, then $\mathcal{E}_{d}$ is a quasi-coarse structure. There are non-symmetric quasi-metrics that induce coarse structures. For example consider the quasi-metric space $(\mathbb{Z}, d)$, where $d$ is defined as follows: for every two points $m, n \in \mathbb{N}$,

$$
d(m, n)=\left\{\begin{array}{lr}
n-m & \text { if } m \leq n \\
2(m-n) & \text { otherwise }
\end{array}\right.
$$

Although $d$ is not symmetric, $\mathcal{E}_{d}$ is a coarse structure.

More examples of entourage spaces will be given in $₫ 2$

An important notion in coarse geometry is boundedness. A subset $A$ of a coarse space $(X, \mathcal{E})$ is called bounded if it satisfies one of the following equivalent properties:

(B1) there exists $x \in A$ and $E \in \mathcal{E}$ such that $A \subseteq E[x]$;

(B2) for every $x \in A$, there exists $E_{x} \in \mathcal{E}$ such that $A \subseteq E_{x}[x]$;

(B3) there exists $E \in \mathcal{E}$ such that, for every $x \in A, A \subseteq E[x]$ (equivalently, $A \times A \in \mathcal{E}$ ).

However, if $X$ is an entourage space, although the implications (B3) $\rightarrow$ (B2) $\rightarrow$ (B1) hold, (B1)-(B3) are not equivalent anymore as Example 1.3 shows.

Example 1.3. (a) Let $X=\{0,1,2\}$ and consider the semi-coarse structure $\mathcal{E}_{1}=\left(\{(0,1),(0,2), \widehat{(1,0)},(2,0)\} \cup \Delta_{X}\right)$ and the quasi-coarse structure $\mathcal{E}_{2}=\left(\{(0,1) \widehat{(0,2)}\} \cup \Delta_{X}\right)$. Then the whole space $X$ satisfies (B1) in both $\mathcal{E}_{1}$ and $\mathcal{E}_{2}$, but it doesn't satisfy (B2).

(b) Let $X=\mathbb{N}$ and $d$ and $d^{\prime}$ be a semi-pseudometric and a quasi-pseudometric defined as follows: for every $m, n \in \mathbb{N}$,

$$
d(m, n)=\left\{\begin{array}{ll}
0 & \text { if } m=n, \\
\min \{m, n\} & \text { otherwise, }
\end{array} \quad \text { and } \quad d^{\prime}(m, n)= \begin{cases}0 & \text { if } n>m, \\
m-n & \text { otherwise }\end{cases}\right.
$$

Then $X$ satisfies (B2) in both the semi-coarse structure $\mathcal{E}_{d}$ and the quasi-coarse structure $\mathcal{E}_{d^{\prime}}$, but it doesn't satisfy (B3).

An entourage space $(X, \mathcal{E})$ is locally finite if, for every $E \in \mathcal{E}$ and $x \in X, E[x]$ is finite. Moreover, $X$ has bounded geometry if there exists a map $\varphi: \mathcal{E} \rightarrow \mathbb{N}$ such that, for every $E \in \mathcal{E}$ and $x \in X,|E[x]| \leq \varphi(E)$.

Let $(X, \mathcal{E})$ be a locally finite entourage structure. Then a subset $A$ of $X$ satisfies (B2) if and only if it satisfies (B3). In fact, if $X$ is locally finite, then every subset $A$ satisfying (B2) is finite. Hence $E=\bigcup_{x \in A} E_{x} \in \mathcal{E}$ and this entourage shows that $A$ satisfies (B3). 
A family $\left\{A_{i}\right\}_{i \in I}$ of subsets of an entourage space $(X, \mathcal{E})$ is uniformly bounded if there exists $E \in \mathcal{E}$ such that, for every $i \in I$ and every $x \in A_{i}, A_{i} \subseteq E[x]$. In particular, every element of a uniformly bounded family satisfies (B3).

Let $(X, \mathcal{E})$ be an entourage space and let $x$ and $y$ be two points. If $\{(x, y)\} \in \mathcal{E}$, then we write $x \downarrow y$. If there exist $x_{0}=x, x_{1}, \ldots, x_{n-1}, x_{n}=y \in X$ such that $x_{i} \downarrow x_{i+1}$ or $x_{i+1} \downarrow x_{i}$, for every $i=0, \ldots, n-1$, then we write $x$ an $y$.

An entourage space $X$ is connected if, for every $x, y \in X, x \leadsto y$. Moreover, $X$ is strongly connected if, for every $x, y \in X, x \downarrow y$ and $y \downarrow x$. Equivalently, an entourage space $(X, \mathcal{E})$ is strongly connected if and only if $\bigcup \mathcal{E}=X \times X$. Note that a coarse $(X, \mathcal{E})$ space is connected if and only if it is strongly connected. This property was already introduced in 21$]$ in the framework of coarse spaces, so no distinction between them was necessary.

Since the relation $\rightsquigarrow$ is an equivalence relation, for every entourage space $X$ we can consider its connected components, which are its equivalence classes under that relation.

If $(X, \mathcal{E})$ is an entourage space, we say that $(X, \mathcal{E})$ is uniformly connected if there exists $E \in \mathcal{E}$ such that, for every $x, y \in X$, there exists $n \in \mathbb{N}$ such that $(x, y) \in\left(E \cup E^{-1}\right)^{n}$. In this case, $X$ is uniformly connected with parameter $E$.

Example 1.4. One may ask whether there are quasi-coarse spaces that are strongly connected, but they are not semicoarse spaces.

Let $(X, d)$ be a psuedo-metric space and let $h: X \rightarrow \mathbb{R}$ be an arbitrary function. Then the function $d_{h}: X \rightarrow \mathbb{R}_{\geq 0}$, defined by the law

$$
d_{h}(x, y)=\left\{\begin{array}{lr}
d(x, y)+h(y)-h(x) & \text { if } h(y)-h(x) \geq 0 \\
d(x, y) & \text { otherwise }
\end{array}\right.
$$

for every $x, y \in X$, defines a quasi-pseudometric space.

Let now $X=\mathbb{Z}, d$ be the usual euclidean metric, and $h(x)=x^{3}$. Then $\left(\mathbb{Z}, \mathcal{E}_{d_{h}}\right)$ is a quasi-coarse space, since $d_{h}$ is a quasi-metric, and it is strongly connected. However, it is not a coarse space. In fact, for every $R \geq 0$ and every $z \in \mathbb{R}$, $d_{h}(z+R, z)=R$, while $d_{h}(z, z+R)=R\left(1+3 z^{2}+3 z R+R^{2}\right)$, and the latter strongly depends on the point $z$. Hence, even though $\{(z+R, z) \mid z \in \mathbb{Z}\} \subseteq E_{R} \in \mathcal{E}_{d}$, there exists no $S \geq 0$ such that $\{(z, z+R) \mid z \in \mathbb{R}\} \subseteq E_{S}$.

In Example 1.2 we introduced metric entourage structures. We now want to characterise those structures.

Let $(X, \mathcal{E})$ be an entourage structure. Define its cofinality as follows: $\operatorname{cf} \mathcal{E}=\inf \{|\mathcal{B}| \mid \widehat{\mathcal{B}}=\mathcal{E}\}$.

Proposition 1.5. Let $(X, \mathcal{E})$ be an entourage space.

(a) Then there exists an extended semi-positive-definite map $d$ on $X$ such that $\mathcal{E}=\mathcal{E}_{d}$ if and only if cf $\mathcal{E} \leq \omega$.

(b) Suppose that $\mathcal{E}$ is a semi-coarse structure. Then there exists an extended semi-metric map $d$ on $X$ such that $\mathcal{E}=\mathcal{E}_{d}$ if and only if $\mathrm{cf} \mathcal{E} \leq \omega$.

Proof. First of all, the "only if" implications in both items (a) and (b) are trivial since $\left\{E_{n} \mid n \in \mathbb{N}\right\}$ is a base of $\mathcal{E}_{d}$.

$(\mathrm{a}, \leftarrow)$ Let $\left\{F_{n} \mid n \in \mathbb{N}\right\}$ be a countable base of $\mathcal{E}$, and, without loss of generality, we can ask that $F_{0}=\Delta_{X}$ and $F_{n} \subseteq F_{n+1}$, for every $n \in \mathbb{N}$. Then define a map $d: X \times X \rightarrow \mathbb{N}$ as follows: for every $x, y \in X$,

$$
d(x, y)=\left\{\begin{array}{lr}
\min \left\{n \mid y \in F_{n}[x]\right\} & \text { if it exists } \\
\infty & \text { otherwise }
\end{array}\right.
$$

It is easy to check that $d$ satisfies the required properties.

$(\mathrm{b}, \leftarrow)$ Suppose that $\mathcal{E}$ is a semi-coarse structure with $\operatorname{cf} \mathcal{E} \leq \omega$. Then we can choose a base $\left\{F_{n} \mid n \in \mathbb{N}\right\}$ as in item (a) with the further property that $E_{n}=E_{n}^{-1}$, for every $n \in \mathbb{N}$. Then the map $d$ as in (3) satisfies the desired properties.

Note that the maps $d$ in Proposition 1.5 are not extended if and only if $(X, \mathcal{E})$ is strongly connected.

The case where the entourage space is a quasi-coarse space (or a coarse space, in particular, which is a classical result) will be discussed in 4.1 .

We can construct the lattice of entourage structures of a set. If $X$ is a set, denote by $\mathfrak{E}(X)$ the family of all entourages structures on $X$. The lattice $\mathfrak{E}(X)$ is ordered by inclusion. More precisely, let $X$ be a set and $\mathcal{E}, \mathcal{E}^{\prime} \in \mathfrak{E}(X)$ be two entourages structures. Then we say that $\mathcal{E}$ is finer that $\mathcal{E}^{\prime}$ if $\mathcal{E} \subseteq \mathcal{E}^{\prime}$ (and $\mathcal{E}^{\prime}$ is coarser than $\mathcal{E}$ ).

Moreover, $\mathfrak{E}(X)$ has a top element $\mathcal{M}_{X}=\mathcal{P}(X \times X)$ (the indiscrete coarse structure) and a minimum element $\mathcal{T}_{X}=\left\{\Delta_{X}\right\}$ (the discrete coarse structure). Finally, $\mathfrak{E}(X)$ is a complete lattice. In fact, for every family $\left\{\mathcal{E}_{i}\right\}_{i \in I}$ of entourage structures, $\bigcap_{i} \mathcal{E}_{i}$ is an entourage structure and so their meet $\bigwedge_{i} \mathcal{E}_{i}$. Moreover, if $\mathcal{E}_{i}$ is a semi-coarse structure (quasi-coarse structure), for every $i \in I$, then also $\bigcap_{i} \mathcal{E}_{i}$ is a semi-coarse structure (quasi-coarse structure, respectively). Hence, the join of a family of entourage structures (semi-coarse structures, quasi-coarse structures, coarse structures) $\left\{\mathcal{E}_{i}\right\}_{i \in I}$ on a set $X$ can be defined as the entourage structure $\bigvee_{i} \mathcal{E}_{i}$ (semi-coarse structure, quasi-coarse structure, coarse structure, respectively) generated by $\bigcup_{i} \mathcal{E}_{i}$, i.e., the finest structure that contains $\mathcal{E}_{i}$, for every $i \in I$.

\subsection{Morphisms between entourage spaces}

Let us now introduce the morphisms between those spaces. Let $f: X \rightarrow Y$ be a map between sets. Denote by $f \times f: X \times X \rightarrow Y \times Y$ the map defined by the law $(f \times f)(x, y)=(f(x), f(y))$, for every $(x, y) \in X \times X$. A map $f:\left(X, \mathcal{E}_{X}\right) \rightarrow\left(Y, \mathcal{E}_{Y}\right)$ between entourage spaces is said to be 
- bornologous (or coarsely uniform, coarse) if $(f \times f)(E) \in \mathcal{E}_{Y}$, for every $E \in \mathcal{E}_{X}$;

- weakly uniformly bounded copreserving if, for every $E \in \mathcal{E}_{Y}$, there exists $F \in \mathcal{E}_{X}$ such that $(f \times f)(F)=E \cap(f(X) \times$ $f(X))$;

- uniformly bounded copreserving if, for every $E \in \mathcal{E}_{Y}$, there exists $F \in \mathcal{E}_{X}$ such that, for every $x \in X, E[f(x)] \cap f(X) \subseteq$ $f(F[x])$

- effectively proper if, for every $E \in \mathcal{E}_{Y},(f \times f)^{-1}(E) \in \mathcal{E}_{X}$.

Note that all the previous properties can be checked just for all the entourages that belong to some bases of the entourage structures.

Proposition 1.6. Let $f:\left(X, \mathcal{E}_{X}\right) \rightarrow\left(Y, \mathcal{E}_{Y}\right)$ be a map between entourage spaces. Then:

(a) if $f$ is effectively proper, then $f$ is uniformly bounded copreserving;

(b) if $f$ is uniformly bounded copreserving, then $f$ is uniformly weakly bounded copreserving.

Proof. (a) Suppose that $f$ is effectively proper and let $E \in \mathcal{E}_{Y}$. Then, for every $x \in X, E[f(x)] \cap f(X) \subseteq f((f \times$ $\left.f)^{-1}(E)[x]\right)$. In fact, for every $y \in X$ such that $(f(x), f(y)) \in E,(x, y) \in(f \times f)^{-1}(E)$ and so $f(y) \in f\left((f \times f)^{-1}(E)[x]\right)$.

(b) Suppose now that $f$ is uniformly bounded copreserving and let $E \in \mathcal{E}_{Y}$. Let $F \in \mathcal{E}_{X}$ be an entourage such that, for every $x \in X, E[f(x)] \cap f(X) \subseteq f(F[x])$. We claim that $E \cap(f \times f)(X \times X) \subseteq(f \times f)(F)$. Let $(x, y) \in E \cap(f \times f)(X \times X)$. There exists $z \in f^{-1}(x)$, and so $y \in E[f(z)] \cap f(X)$, which implies that there exists $w \in F[z] \cap f^{-1}(y)$. Finally, note that $(z, w) \in F$ and $(x, y)=(f(z), f(w)) \in(f \times f)(F)$.

If $f$ is injective, then both implications of Proposition 1.6 can be easily reverted. Proposition 1.7 gives another condition that implies their reversibility.

Note that a map $f:\left(X, \mathcal{E}_{X}\right) \rightarrow Y$ from an entourage space to a set has uniformly bounded fibers if and only if $R_{f}=\{(x, y) \in X \times X \mid f(x)=f(y)\} \in \mathcal{E}_{X}$. We call such a map large-scale injective.

Proposition 1.7. Let $f:\left(X, \mathcal{E}_{X}\right) \rightarrow\left(Y, \mathcal{E}_{Y}\right)$ be a map between entourage spaces. If $f$ is effectively proper, then $f$ is large-scale injective. Moreover, if $\mathcal{E}_{X}$ is a quasi-coarse structure, then the following properties are equivalent:

(a) $f$ is large-scale injective and it is weakly uniformly bounded copreserving;

(b) $f$ is large-scale injective and it is uniformly bounded copreserving;

(c) $f$ is effectively proper.

Proof. The first statement can be easily proved: since $\Delta_{Y} \in \mathcal{E}_{Y}$, then $R_{f}=(f \times f)^{-1}\left(\Delta_{Y}\right) \in \mathcal{E}_{X}$.

In view of Proposition 1.6, we just need to show the implication $(\mathrm{a}) \rightarrow(\mathrm{c})$. Suppose now that $f$ is weakly uniformly bounded copreserving and $R_{f} \in \mathcal{E}_{X}$. Let $E \in \mathcal{E}_{Y}$ and $(x, y)$ be an arbitrary point in $(f \times f)^{-1}(E)$. Let $F \in \mathcal{E}_{X}$ such that $(f \times f)(F)=E \cap(f(X) \times f(X))$. Then there exists $(z, w) \in F$ such that $(f(x), f(y))=(f(z), f(w))$ and thus

$$
(x, y)=(x, z) \circ(z, w) \circ(w, y) \in R_{f} \circ F \circ R_{f} \in \mathcal{E}_{X} .
$$

Proposition 1.8. Let $f:\left(X, \mathcal{E}_{X}\right) \rightarrow\left(Y, \mathcal{E}_{Y}\right)$ be a uniformly bounded copreserving surjective map between entourage spaces. Then $Y$ has bounded geometry ( $Y$ is locally finite) whenever $X$ has bounded geometry ( $X$ is locally finite, respectively).

Proof. Suppose that $\varphi: \mathcal{E}_{X} \rightarrow \mathbb{N}$ is a map that demonstrates that $\left(X, \mathcal{E}_{X}\right)$ has bounded geometry. Let $E \in \mathcal{E}_{Y}$. Then there exists $F \in \mathcal{E}_{X}$ such that, for every $x \in X, E[f(x)] \subseteq f(F[x])$. Hence, $|E[f(x)]| \leq|F[x]| \leq \varphi(F)$. The other implication can be similarly proved.

A bijective map $f:\left(X, \mathcal{E}_{X}\right) \rightarrow\left(Y, \mathcal{E}_{Y}\right)$ between entourage spaces is called an asymorphism if it satisfies the following, equivalent, properties:

- $f$ and $f^{-1}$ are bornologous;

- $f$ is bornologous and weakly uniformly bounded copreserving;

- $f$ is bornologous and uniformly bounded copreserving;

- $f$ is bornologous and effectively proper.

Let $\left(X, \mathcal{E}_{X}\right)$ and $\left(Y, \mathcal{E}_{Y}\right)$ be two asymorphic entourage spaces. Then $\mathcal{E}_{X}$ is a semi-coarse structure (quasi-coarse structure) if and only if $\mathcal{E}_{Y}$ is a semi-coarse structure (quasi-coarse structure, respectively). For the proof of this fact, we address to [17, where the authors used the equivalent approach through ball structures (see $\$ 1.2$ for the introduction of these structures).

Furthermore, if $X$ and $Y$ are two asymorphic entourage spaces, then $X$ is (strongly or uniformly) connected if and only if $Y$ is (respectively strongly or uniformly) connected.

\subsection{Approach via ball structures}

Let $(X, \mathcal{E})$ be an entourage structure. Then we can associate to it a triple $\mathfrak{B}_{\mathcal{E}}=\left(X, P, B_{\mathcal{E}}\right)$, where $P=\{E \in \mathcal{E} \mid$ $\left.\Delta_{X} \subseteq E\right\}$ and $B_{\mathcal{E}}(x, E)=E[x]$, for every $x \in X$ and every $E \in P$. It is an example of ball structure. 
Definition 1.9. ([17, 20]) A ball structure is a triple $\mathfrak{B}=(X, P, B)$ where $X$ and $P$ are sets, $P \neq \emptyset$, and $B: X \times P \rightarrow \mathcal{P}(X)$ is a map, such that $x \in B(x, r)$ for every $x \in X$ and every $r \in P$. The set $X$ is called support of the ball structure, $P-$ set of radii, and $B(x, r)$ - ball of center $x$ and radius $r$. In case $X=\emptyset$, the map $B$ is the empty map.

The terminology and the intuition come from the metric setting: if $(X, d)$ is a metric space, then $\mathfrak{B}_{d}=\left(X, \mathbb{R}_{\geq 0}, B_{d}\right)$, where $B_{d}(x, R)$ is the closed ball centred in $x \in X$ with radius $R \geq 0$, is a ball structure.

For a ball structure $(X, P, B), x \in X, r \in P$ and a subset $A$ of $X$, one puts

$$
B^{*}(x, r)=\{y \in X \mid x \in B(y, r)\} \quad B(A, r)=\bigcup\{B(x, r) \mid x \in A\} .
$$

A ball structure $\mathfrak{B}=(X, P, B)$ is said to be:

- weakly upper multiplicative if, for every pair of radii $r, s \in P$ there exists $t \in P$ such that $B(x, r) \cup B(x, s) \subseteq B(x, t)$, for every $x \in X$;

- upper multiplicative if, for every pair of radii $r, s \in P$ there exists $t \in P$ such that $B(B(x, r), s) \subseteq B(x, t)$, for every $x \in X$

- upper symmetric if, for every pair of radii $r, s \in P$ there exist $r^{\prime}, s^{\prime} \in P$ such that $B^{*}(x, r) \subseteq B\left(x, r^{\prime}\right)$ and $B(x, s) \subseteq$ $B^{*}\left(x, s^{\prime}\right)$, for every $x \in X$.

It is trivial that upper multiplicativity implies weak upper multiplicativity since every ball contains its center.

Definition 1.10. A ball structure is

- a semi-ballean if it is weakly upper multiplicative and upper symmetric;

- a quasi-ballean if it is upper multiplicative;

- a ballean ([17]) if it is both a semi-ballean and a quasi-ballean.

For every entourage space $(X, \mathcal{E}), \mathfrak{B}_{\mathcal{E}}$ is indeed a weakly upper multiplicative ball structure. Moreover, if $\mathcal{E}$ is a semi-coarse structure, then $\mathfrak{B}_{\mathcal{E}}$ is a semi-ballean, while, if $\mathcal{E}$ is a quasi-coarse structure, then $\mathfrak{B}_{\mathcal{E}}$ is a quasi-ballean.

We have seen how we construct ball structures from entourage structures. Let us now discuss the opposite construction. Let $\mathfrak{B}=(X, P, B)$ be a weakly multiplicative ball structure. Then we can define an associated entourage structure $\mathcal{E}_{\mathfrak{B}}$ of $X$ as follows: for every $r \in P$,

$$
E_{r}=\bigcup_{x \in X}(\{x\} \times B(x, r))
$$

and the family $\left\{E_{r} \mid r \in P\right\}$ is a base for the entourage structure $\mathcal{E}_{\mathfrak{B}}$. Moreover,

- if $\mathfrak{B}$ is a semi-ballean, then $\mathcal{E}_{\mathfrak{B}}$ is a semi-coarse structure;

- if $\mathfrak{B}$ is a quasi-ballean, then $\mathcal{E}_{\mathfrak{B}}$ is a quasi-coarse structure;

- if $\mathfrak{B}$ is a ballean, then $\mathcal{E}_{\mathfrak{B}}$ is a coarse structure.

Let $\mathfrak{B}$ and $\mathfrak{B}^{\prime}$ be two weakly multiplicative ball structure on the same support $X$. Then we identify those two ball structure, and we write $\mathfrak{B}=\mathfrak{B}^{\prime}$, if $\mathcal{E}_{\mathfrak{B}}=\mathcal{E}_{\mathfrak{B}^{\prime}}$. We soon give a characterization of the equality between ball structures. Hence, for every entourage space $(X, \mathcal{E})$ and every weakly multiplicative ball structure $\mathfrak{B}$ on $X$,

$$
\mathcal{E}_{\mathfrak{B} \mathcal{E}}=\mathcal{E} \quad \text { and } \quad \mathfrak{B}_{\mathcal{E}_{\mathfrak{B}}}=\mathfrak{B}
$$

The equivalence between coarse structures and balleans have already been widely discussed (see, for example, [20, 6]).

Let $\mathfrak{B}=\left(X, P_{X}, B_{X}\right)$ and $\mathfrak{B}_{Y}=\left(Y, P_{Y}, B_{Y}\right)$ be two weakly multiplicative ball structures and $f: \mathfrak{B}_{X} \rightarrow \mathfrak{B}_{Y}$ be a map. The map $f$ is bornologous if the following equivalent properties are fulfilled:

- $f:\left(X, \mathcal{E}_{\mathfrak{B}_{X}}\right) \rightarrow\left(Y, \mathcal{E}_{\mathfrak{B}_{Y}}\right)$ is bornologous;

- for every radius $r \in P_{X}$, there exists $s \in P_{Y}$ such that $f\left(B_{X}(x, r)\right) \subseteq B_{Y}(f(x), s)$, for every $x \in X$.

Similarly, $f$ is uniformly bounded copreserving if the following equivalent properties are satisfies:

- $f:\left(X, \mathcal{E}_{\mathfrak{B}_{X}}\right) \rightarrow\left(Y, \mathcal{E}_{\mathfrak{B}_{Y}}\right)$ is uniformly bounded copreserving;

- for every $s \in P_{Y}$, there exists $r \in P_{X}$ such that $B_{Y}(f(x), s) \cap f(X) \subseteq f\left(B_{X}(x, r)\right)$, for every $x \in X$.

Thanks to this characterisation of being uniformly bounded copreserving, it is clear that this notion generalises the one of $\succ$-mapping $([20])$ : the map $f$ is a $\succ$-mapping if, for every $s \in P_{Y}$, there exists $r \in P_{X}$ such that $B_{Y}(f(x), s) \subseteq$ $f\left(B_{X}(x, r)\right)$, for every $x \in X$. Of course, a surjective map is uniformly bounded copreserving if and only if it is a $\succ$-mapping. However, the second definition is very restrictive when the map is not surjective. In fact, if a map $f:\left(X, \mathcal{E}_{X}\right) \rightarrow\left(Y, \mathcal{E}_{Y}\right)$ is a $\succ$-mapping, then, if $(f(x), y) \in E$ for some $E \in \mathcal{E}_{Y}, x \in X$ and $y \in Y$, then $y \in f(X)$.

Finally, let us give the promised characterisation of the equality between ball structures on the same support. If $\mathfrak{B}$ and $\mathfrak{B}^{\prime}$ are two ball structure on a set $X$, then $\mathfrak{B}=\mathfrak{B}^{\prime}$ if and only if $i d_{X}: \mathfrak{B} \rightarrow \mathfrak{B}^{\prime}$ is an asymorphism, i.e., bornologous with also its inverse bornologous.

We have briefly recalled how coarse spaces and balleans are equivalent constructions. In the literature, there is a third way to describe coarse spaces by using coverings: the so-called large-scale structures ([7], also know as asymptotic proximities in [16]). Those are large-scale counterpart of the classical approach to uniformities via coverings (see [10]). Moreover, in [15], the authors presented a way to use the covering approach to describe quasi-uniformities. Hence the following question naturally arises.

Question 1.11. Is it possible to give a characterisation of entourage structures, semi-coarse structures or quasi-coarse structures through coverings? 


\section{Some examples of entourage spaces}

In this section we enlist some examples of entourage spaces.

\subsection{Relation entourage structures}

Let $X$ be a set and $\mathscr{R}$ be a reflexive relation of $X$. Define $\mathcal{E}_{\mathscr{R}}=\widehat{\{\mathscr{R}\}}$, which is an entourage structure, called relation entourage structure. Moreover, $\mathscr{R}$ is symmetric if and only if $\mathcal{E}_{\mathscr{R}}$ is a semi-coarse structure, while $\mathscr{R}$ is transitive if and only if $\mathcal{E}_{\mathscr{R}}$ is a quasi-coarse structure. Furthermore, note that, $\left(\mathcal{E}_{\mathscr{R}}\right)^{-1}=\mathcal{E}_{\mathscr{R}-1}$, where $\mathscr{R}^{-1}$ denotes the inverse of $\mathscr{R}$ as an entourage. Another entourage structure that can be defined from a reflexive relation $\mathscr{R}$ on a set $X$ is the following: $\mathcal{E}_{\mathscr{R}}^{\text {fin }}=[\mathscr{R}]^{<\infty} \cup\left\{\Delta_{X}\right\}$.

It is easy to verify the following result.

Proposition 2.1. Let $f:\left(X, \mathscr{R}_{X}\right) \rightarrow\left(Y, \mathscr{R}_{Y}\right)$ be a map between sets endowed with reflexive relations. Then the following properties are equivalent:

(a) $f$ preserves the relation (i.e., for every $x, y \in X, f(x) \mathscr{R}_{Y} f(y)$ provided that $x \mathscr{R}_{X} y$ );

(b) $f:\left(X, \mathcal{E}_{\mathscr{R}_{X}}\right) \rightarrow\left(Y, \mathcal{E}_{\mathscr{R}_{Y}}\right)$ is bornologous;

(c) $f:\left(X,\left(\mathcal{E}_{\mathscr{R}_{X}}\right)^{-1}\right) \rightarrow\left(Y,\left(\mathcal{E}_{\mathscr{R}_{Y}}\right)^{-1}\right)$ is bornologous;

(d) $f:\left(X, \mathcal{E}_{\mathscr{R}_{X}}^{\text {fin }}\right) \rightarrow\left(Y, \mathcal{E}_{\mathscr{R}_{Y}}^{\text {fin }}\right)$ is bornologous;

(e) $f:\left(X,\left(\mathcal{E}_{\mathscr{R}_{X}}^{\text {fin }}\right)^{-1}\right) \rightarrow\left(Y,\left(\mathcal{E}_{\mathscr{R}_{Y}}^{\text {fin }}\right)^{-1}\right)$ is bornologous.

We have discussed how one can construct entourage structures from reflexive relations. Now, we focus on the opposite process. Let $(X, \mathcal{E})$ be an entourage space. Then we define $\mathscr{R}_{\mathcal{E}}=\bigcup \mathcal{E}$, which is a reflexive relation since $\Delta_{X} \in \mathcal{E}$. Moreover, if $\mathcal{E}$ is a semi-coarse structure, then $\mathscr{R}_{\mathcal{E}}$ is symmetric, and, if $\mathcal{E}$ is a quasi-coarse structure, then $\mathscr{R}_{\mathcal{E}}$ is transitive.

Note that, if $\mathscr{R}$ is a reflexive relation on $X$, then

$$
\mathscr{R}=\mathscr{R}_{\mathcal{E}_{\mathscr{R}}}=\mathscr{R}_{\mathcal{E}_{\mathscr{R}}^{f i n}}
$$

Meanwhile, if $(X, \mathcal{E})$ is an entourage space, then

$$
\mathcal{E}_{\mathscr{R}_{\mathcal{E}}}^{\text {fin }} \subseteq \mathcal{E} \subseteq \mathcal{E}_{\mathscr{R}_{\mathcal{E}}}
$$

The inclusions in (4) can be strict. Consider, for example, $\mathbb{R}$ endowed with the usual metric $d$. Then $\mathcal{E}_{\mathscr{R}_{\mathcal{E}_{d}}}^{\text {in }} \mathcal{E}_{d} \subsetneq \mathcal{E}_{\mathscr{R}_{\mathcal{E}_{d}}}$. Furthermore, note that $\mathcal{E}=\mathcal{E}_{\mathscr{R}_{\mathcal{E}}}$ if and only if $\bigcup \mathcal{E} \in \mathcal{E}$ and, thus, every entourage structure $\mathcal{E}$ on a finite set $X$ is a relation entourage structure.

\subsection{Graphic quasi-coarse structures}

Let $\Gamma=(V, E)$ be a directed graph. Define the path extended quasi-metric over $V$ to be the value:

$$
d(v, w)=\left\{\begin{array}{lr}
\min \left\{\left|\left\{\left(x_{i}, x_{i+1}\right)\right\}_{i=0}^{n-1}\right| \mid \forall i=0, \ldots, n-1,\left(x_{i}, x_{i+1}\right) \in E, x_{0}=v, x_{n}=w\right\} & \text { if it exists, } \\
\infty & \text { otherwise. }
\end{array}\right.
$$

This is an extended quasi-metric and $\mathcal{E}_{d}$ is called graphic quasi-coarse structure.

Let The graphic quasi-coarse space can be extended to the points on the graph edges, by identifying every edge with the interval $[0,1]$ endowed to the relation quasi-coarse structure associated to the usual order $\leq$ on $[0,1]$. More precisely, if $\Gamma=(V, E)$ is a directed graph and $(v, w) \in E$, then we identify 0 and $v$ and 1 and $w$, respectively. This new quasi-coarse structure is called extended graphic quasi-coarse structure.

Let $f: \Gamma(V, E) \rightarrow \Gamma^{\prime}\left(V^{\prime}, E^{\prime}\right)$ be a map between oriented graphs. Then $f$ is said to be a graph homomorphism if, for every $(x, y) \in E$, either $f(x)=f(y)$ or $(f(x), f(y)) \in E^{\prime}$. If $f: \Gamma(V, E) \rightarrow \Gamma^{\prime}\left(V^{\prime}, E^{\prime}\right)$ is a graph homomorphism, then $f$ sends directed paths into non-longer directed paths. Hence $f:(V, d) \rightarrow\left(V^{\prime}, d\right)$ is non-expanding (i.e., $d(f(x), f(y)) \leq$ $d(x, y)$, for every $x, y \in V)$, and thus $f:\left(V, \mathcal{E}_{d}\right) \rightarrow\left(V^{\prime}, \mathcal{E}_{d}\right)$ is bornologous.

\section{$2.3 \quad$ Entourage hyperstructures}

Let $(X, \mathcal{E})$ be an entourage structure. We define the following two entourage structures on $\mathcal{P}(X)$ :

$$
\mathcal{H}(\mathcal{E})=\left\{\mathcal{H}(E) \mid \widehat{\Delta_{X} \subseteq} E \in \mathcal{E}\right\} \quad \text { and } \quad \exp \mathcal{E}=\left\{\exp E \mid \widehat{\Delta_{X} \subseteq} E \in \mathcal{E}\right\}=\mathcal{H}(\mathcal{E}) \cap \mathcal{H}(\mathcal{E})^{-1},
$$

where, for every $E \in \mathcal{E}$,

$$
\mathcal{H}(E)=\{(A, B) \mid B \subseteq E[A]\} \quad \text { and } \quad \exp (E)=\mathcal{H}(E) \cap \mathcal{H}(E)^{-1}
$$


named entourage hyperstructure and semi-coarse hyperstructure, respectively. The way we obtained semi-coarse hyperstructures from entourage hyperstructures will be generalised in 3.1 . In 4, the authors defined the notion of hyperballean. It is an equivalent way to define the semi-coarse hyperspace of a coarse space in terms of balleans. Moreover, hyperballeans already appeared in [19], although, the authors just considered the subspace of the hyperballean whose support is the family of all non-empty bounded subsets.

First of all, note that, if $\mathcal{E}$ is an entourage structure, then both $\mathcal{H}(\mathcal{E})$ and $\exp \mathcal{E}$ are entourage structures since $\mathcal{H}(E) \cap \mathcal{H}(F)=\mathcal{H}(E \cup F)$, for every $E, F \in \mathcal{E}$. More precisely, exp $\mathcal{E}$ is actually a semi-coarse structure. Furthermore, if $\mathcal{E}$ is quasi-coarse structure, then $\mathcal{H}(\mathcal{E})$ is a quasi-coarse structure, while exp $\mathcal{E}$ is a coarse structure. In fact, for every $E, F \in \mathcal{E}$, if $(A, B) \circ(B, C) \in \mathcal{H}(E) \circ \mathcal{H}(F)$, then $B \subseteq E[A]$ and $C \subseteq F[B]$, which implies that $C \subseteq F[E[A]]=$ $(F \circ E)[A]$ and so $(A, C) \in \mathcal{H}(F \circ E)$. Note that $\mathcal{H}(\mathcal{E})$ is not a semi-coarse structure, unless the support $X$ of $\mathcal{E}$ is empty: in fact, $(X, \emptyset) \in \mathcal{H}\left(\Delta_{X}\right)$, although, for every $E \in \mathcal{E}, E[\emptyset]=\emptyset$. Moreover, even if we consider the subspace $\left(\mathcal{P}(X) \backslash\{\emptyset\},\left.\mathcal{H}(\mathcal{E})\right|_{\mathcal{P}(X) \backslash\{\emptyset\}}\right)$, it is a semi-coarse structure if and only if $X$ satisfies (B3). In fact, $(X,\{x\}) \in \mathcal{H}\left(\Delta_{X}\right)$, for every $x \in X$.

Every map $f: X \rightarrow Y$ between sets can be extended to a map $\bar{f}: \mathcal{P}(X) \rightarrow \mathcal{P}(Y)$ such that, for every $A \in \mathcal{P}(X)$, $\bar{f}(A)=f(A) \in \mathcal{P}(Y)$.

Proposition 2.2. Let $f:\left(X, \mathcal{E}_{X}\right) \rightarrow\left(Y, \mathcal{E}_{Y}\right)$ be a between entourage spaces. The following properties are equivalent:

(a) $f:\left(X, \mathcal{E}_{X}\right) \rightarrow\left(Y, \mathcal{E}_{Y}\right)$ is bornologous;

(b) $\bar{f}:\left(\mathcal{P}(X), \mathcal{H}\left(\mathcal{E}_{X}\right)\right) \rightarrow\left(\mathcal{P}(Y), \mathcal{H}\left(\mathcal{E}_{Y}\right)\right)$ is bornologous.

Proof. As for the implication $(\mathrm{a}) \rightarrow(\mathrm{b})$, if $f$ is bornologous, then the inclusion $(\bar{f} \times \bar{f})(\mathcal{H}(E)) \subseteq \mathcal{H}((f \times f)(E))$, for every $E \in \mathcal{E}_{X}$, holds, and the thesis follows. Conversely, (b) $\rightarrow($ a) is a consequence of the fact that, for every entourage space $\left(Z, \mathcal{E}_{Z}\right)$, if $E \in \mathcal{E}_{Z}$ and $x, y \in Z$, then $(x, y) \in E$ if and only if $(\{x\},\{y\}) \in \mathcal{H}(E)$.

We conclude this discussion by very briefly relating to the classical theory of quasi-uniform spaces. The definition of a quasi-uniform structure on the power set of a quasi-uniform space is a classical construction, and it is very similar to the entourage hyperstructure we have just introduced. A wide introduction and a broad bibliography can be found in 12. Moreover, we refer to [10] for an analogue of the semi-coarse hyperstructure in the framework of uniform spaces.

\subsection{Finitely generated monoids}

A magma is a pair $(M, \cdot)$, where $M$ is a set and $:: M \times M \rightarrow M$ is a map. A magma $(M, \cdot)$ is called unitary if there exists a neutral element $e \in M$ such that $g \cdot e=e \cdot g=g$, for every $g \in M$. A unitary magma is a monoid, if · is associative.

Let $M$ be a monoid. We say that $M$ is finitely generated if there exists a finite subset $\Sigma$ of $M$ such that, for every $g \in M$ there exist $n \in \mathbb{N}$ and $\sigma_{1}, \ldots, \sigma_{n} \in \Sigma$ which satisfy $g=\sigma_{1} \cdots \sigma_{n}$.

In this subsection we want to briefly discuss the existence of precisely two inner quasi-coarse structures on a finitely generated monoid (see Proposition 2.3). The proof we give is similar to the case of finitely generated groups (see, for example, [8]).

Let $M$ be a monoid which is finitely generated by $\Sigma$. Let us define the (left) word extended quasi-metric $d_{\Sigma}^{\lambda}$ as follows: for every pair of elements $x, y \in M$,

$$
d_{\Sigma}^{\lambda}(x, y)=\min \left\{n \mid \exists \sigma_{1}, \ldots, \sigma_{n} \in \Sigma: y=x \sigma_{1} \cdots \sigma_{n}\right\}
$$

(we denote $\min \emptyset=\infty$ ). The map $d_{\Sigma}^{\lambda}: M \times M \rightarrow \mathbb{N} \cup\{\infty\}$ is actually an extended quasi-metric and thus $M$ can be endowed with the metric entourage structure $\mathcal{E}_{d_{\Sigma}^{\lambda}}$, which is a quasi-coarse structure. Moreover, note that $d_{\Sigma}^{\lambda}$ is left-nonexpanding, i.e., for every $x, y, z \in M, d_{\Sigma}^{\lambda}(z x, z y) \leq d_{\Sigma}^{\lambda}(x, y)$. If $M$ is a finitely generated group, then $d_{\Sigma}^{\lambda}$ is left-invariant (i.e., for every $\left.x, y, z \in M, d_{\Sigma}^{\lambda}(z x, z y)=d_{\Sigma}^{\lambda}(x, y)\right)$. Similarly, one can define a right-non-expanding extended quasi-metric $d_{\Sigma}^{\rho}$ on $M$, called (right) word quasi-metric: for every $x, y \in M$,

$$
d_{\Sigma}^{\rho}(x, y)=\min \left\{n \mid \exists \sigma_{1}, \ldots, \sigma_{n} \in \Sigma: y=\sigma_{1} \cdots \sigma_{n} x\right\}
$$

Proposition 2.3. Let $M$ be a monoid and $\Sigma$ and $\Delta$ be two finite subsets of $M$ which generate the whole monoid. Then $\mathcal{E}_{d_{\Sigma}^{\lambda}}=\mathcal{E}_{d_{\Delta}^{\lambda}}$ and $\mathcal{E}_{d_{\Sigma}^{\rho}}=\mathcal{E}_{d_{\Delta}^{\rho}}$

Proof. Define $k=\max \left\{d_{\Delta}^{\lambda}(e, \sigma) \mid \sigma \in \Sigma\right\}$ and $l=\max \left\{d_{\Sigma}^{\lambda}(e, \delta) \mid \delta \in \Delta\right\}$. Let $x, y \in M$, suppose that $d_{\Sigma}^{\lambda}(x, y)=n$ and let $\sigma_{1}, \ldots, \sigma_{n} \in \Sigma$ such that $y=x \sigma_{1} \cdots \sigma_{n}$. Suppose that $\sigma_{i}=\delta_{i, 1} \cdots \delta_{i, k_{i}}$, for every $i=1, \ldots, n$, where $k_{i}=d_{\Delta}^{\lambda}\left(e, \sigma_{i}\right)$ and $\delta_{i, j} \in \Delta$, for every $i=1, \ldots, n$ and $j=1, \ldots, k_{i}$. Then

$$
y=x \sigma_{1} \cdots \sigma_{n}=x \delta_{1,1} \cdots \delta_{1, k_{1}} \delta_{2,1} \cdots \delta_{n, k_{n}}
$$

and so $d_{\Delta}^{\lambda}(x, y) \leq \sum_{i=1}^{n} k_{i} \leq n k=k d_{\Sigma}^{\lambda}(x, y)$. Hence, $\mathcal{E}_{d_{\Sigma}^{\lambda}} \subseteq \mathcal{E}_{d_{\Delta}^{\lambda}}$. Similarly, $d_{\Sigma}^{\lambda}(x, y) \leq l d_{\Delta}^{\lambda}(x, y)$ and then $\mathcal{E}_{d_{\Delta}^{\lambda}} \subseteq \mathcal{E}_{d_{\Sigma}^{\lambda}}$. A similar proof shows that $\mathcal{E}_{d_{\Sigma}^{\rho}}=\mathcal{E}_{d_{\Delta}^{\rho}}$. 
It is possible to extend the notion of Cayley graph, which is a useful tool to represent a finitely generated group, in the framework of finitely generated monoids. Let $M$ be a monoid and $\Sigma \subseteq M$ a finite subset which generates $M$. Then the (left) Cayley graph of $M$ associated to $\Sigma$ is the directed graph $\operatorname{Cay}^{\lambda}(M, \Sigma)=(M, E)$, where $(x, y) \in E$ if and only if there exists $\sigma \in \Sigma$ such that $y=x \sigma$ or, equivalently, $d_{\Sigma}^{\lambda}(x, y)=1$. Similarly $\operatorname{Cay}^{\rho}(M, \Sigma)$, the (right) Cayley graph, can be constructed. The quasi-coarse space $\left(M, \mathcal{E}_{d_{\Sigma}^{\lambda}}\right)$ and the graphic quasi-coarse structure on $\operatorname{Cay}^{\lambda}(M, \Sigma)$ (see 92.2$)$ are asymorphic. Similarly, $\left(M, \mathcal{E}_{d_{\Sigma}^{\rho}}\right)$ and the graphic quasi-coarse structure on $\operatorname{Cay}^{\rho}(M, \Sigma)$ are asymorphic.

\subsection{Entourage structures on certain algebraic structures}

In the classical coarse geometry, the geometry on finitely generated groups introduced via word metrics can be generalised to groups which are not finitely generated (see, for example, 18, 14]). In this subsection we want to give a quick presentation of ways to introduce entourage structures on particular, more general algebraic structures.

A unitary magma $(M, \cdot)$ is a loop if, for every $a, b \in M$ there exist a unique $x \in M$ and a unique $y \in M$ such that

$$
a \cdot x=b \quad \text { and } \quad y \cdot a=b .
$$

Since $e \cdot e=e$, (5) implies that $e$ is the only neutral element. By (5), for every $g \in M$, there exist two elements $g^{\rho}, g^{\lambda} \in M$ such that $g \cdot g^{\rho}=e$ and $g^{\lambda} \cdot g=e$. Note that $\left(g^{\rho}\right)^{\lambda}=\left(g^{\lambda}\right)^{\rho}=g$, for every $g \in M$ (in fact, $\left(g^{\rho}\right)^{\lambda} \cdot g^{\rho}=e, g^{\lambda}\left(g^{\lambda}\right)^{\rho}=e$, $g \cdot g^{\rho}=e$, and $g^{\lambda} \cdot g=e$ and the conclusions follow by uniqueness of the solution of (50) ). A loop $(M, \cdot)$ has right inverse property if, for every $g, h \in M,(g \cdot h) \cdot h^{\rho}=g$. Similarly, a loop $(M, \cdot)$ has left inverse property if, for every $g, h \in M$, $g^{\lambda} \cdot(g \cdot h)=h$. A loop has inverse property if it has both right and left inverse property. A loop $M$ is said to have two-side inverses if $g^{\lambda}=g^{\rho}$, for every $g \in M$, and, in this case, we denote the inverse of $g$ by $g^{-1}$.

A unitary submagma $N$ of a unitary magma $M$ is a subset $N \subseteq M$ that contains the identity of $M$ and it is closed under the operation. A unitary submagma $N$ is a subloop of a loop $M$ if, for every parameters in $N$, the solutions of (5) belongs to $N$. A unitary submagma $N$ of a monoid $M$ is called a submonoid.

Definition 2.4. Let $M$ be an unitary magma and $\mathcal{I}$ be a family of subsets of $M$.

- $\mathcal{I}$ is a magmatic ideal if it is an ideal on $M$ such that $\{e\} \in \mathcal{I}$ and, for every $\{x\},\{y\} \in \mathcal{I},\{x y\} \in \mathcal{I}$.

- If $M$ is a monoid, $\mathcal{I}$ is a monoid ideal if it is a magmatic ideal and, for every $H, K \in \mathcal{I}, H \cdot K=\{h \cdot k \mid h \in H, k \in K\} \in \mathcal{I}$.

- If $M$ is a loop, a magmatic ideal $\mathcal{I}$ is a right loop ideal if, for every $F \in \mathcal{I}, F^{\rho}=\left\{g^{\rho} \mid g \in F\right\} \in \mathcal{I}$, I is a left loop ideal if, for every $F \in \mathcal{I}, F^{\lambda}=\left\{g^{\lambda} \mid g \in F\right\} \in \mathcal{I}$, and $\mathcal{I}$ is a loop ideal if it is both a left loop ideal and a right loop ideal.

- If $M$ is a group, $\mathcal{I}$ is a group ideal ([20]) if it is both a monoid ideal and a loop ideal.

If $\mathcal{I}$ is a magmatic ideal on a unitary magma $M$, then $\bigcup \mathcal{I}$ is a unitary submagma. Similarly, $\bigcup \mathcal{I}$ is a subloop (a submonoid, or a subgroup) if $\mathcal{I}$ is a loop ideal (a monoid ideal, or a group ideal, respectively). Moreover, if $\mathcal{I}$ is a right loop ideal on a loop with right inverse property (a left loop ideal on a loop with left inverse property), then $\bigcup \mathcal{I}$ has right inverse property (left inverse property, respectively).

The leading examples of magmatic ideals are the finitary magmatic ideal, the finitary monoid ideal, the finitary (left, right) loop ideal, and the finitary group ideal.

Example 2.5. Let $M$ be an unitary magma, then the family $\mathcal{I}=[M]^{<\infty}=\{F \subseteq M \mid M$ is finite $\}$ is a magmatic ideal, called finitary magmatic ideal. If $M$ is a monoid, then $\mathcal{I}$ is a monoid ideal, called finitary monoid ideal. If $M$ is a loop with (right) inverse property, then $\mathcal{I}$ is a (right) loop ideal, called finitary (right) loop ideal. Finally, if $M$ is a group, then $\mathcal{I}$ is a group ideal, called finitary group ideal.

Let $M$ be a unitary magma and $\mathcal{I}$ a magmatic ideal on $M$. If $A$ is a subset of $M \times M$, define $M \cdot A=\{(m x, m y) \mid$ $m \in M,(x, y) \in A\}$. Then we can define the left magmatic entourage structure $\mathcal{E}_{\mathcal{I}}$ on $M$ as follows:

$$
\mathcal{E}_{\mathcal{I}}^{\lambda}=\left\{E_{I}^{\lambda \mid I \in} \mathcal{I}\right\}, \quad \text { where, for every } I \in \mathcal{I}, \quad E_{I}^{\lambda}=M(\{e\} \times I)=\{(x, x k) \mid x \in M, k \in I\} .
$$

Similarly, we can define the right magmatic entourage structure $\mathcal{E}_{\mathcal{I}}^{\rho}$, where the action of $M$ is on the right:

$$
\mathcal{E}_{\mathcal{I}}^{\rho}=\left\{E_{I}^{\widehat{\rho} \mid \widehat{I} \in \mathcal{I}\}}, \quad \text { where, for every } I \in \mathcal{I}, \quad E_{I}^{\rho}=(\{e\} \times I) M .\right.
$$

Proposition 2.6. Let $M$ be a unitary magma and $\mathcal{I}$ a magmatic ideal on $M$.

(a) $\mathcal{E}_{\mathcal{I}}^{\lambda}$ and $\mathcal{E}_{\mathcal{I}}^{\rho}$ are entourage structures.

(b) If $M$ is a loop with right inverse property and $\mathcal{I}$ is a right loop ideal (with the left inverse property and $\mathcal{I}$ is a left loop ideal), then $\mathcal{E}_{\mathcal{I}}^{\lambda}$ is a semi-coarse structure, called left loop semi-coarse structure ( $\mathcal{E}_{\mathcal{I}}^{\rho}$ is a semi-coarse structure, called right loop semi-coarse structure, respectively).

(c) If $M$ is a monoid and $\mathcal{I}$ is a monoid ideal, then $\mathcal{E}_{\mathcal{I}}^{\lambda}$ and $\mathcal{E}_{\mathcal{I}}^{\rho}$ are quasi-coarse structures, called left monoid quasi-coarse structure and right monoid quasi-coarse structure, respectively.

(d) If $M$ is a group, then $\mathcal{E}_{\mathcal{I}}^{\lambda}$ and $\mathcal{E}_{\mathcal{I}}^{\rho}$ are coarse structures, called left group coarse structure and right group coarse structure, respectively. 
Proof. Item (a) is trivial and item (d) has already been proved ([14]).

(b) Let $M$ be a loop with right inverse property and $\mathcal{I}$ be a right loop. Let $K \in \mathcal{I}$. Then, for every $(x, x k) \in E_{K}^{\lambda}$, where $x \in M$ and $k \in K,(x k, x)=\left(x k,(x k) k^{\rho}\right) \in E_{K^{\rho}}^{\lambda}$. Hence $\left(E_{K}^{\lambda}\right)^{-1} \subseteq E_{K^{\rho}}^{\lambda} \in \mathcal{E}_{\mathcal{I}}^{\lambda}$. We can prove similarly the other assertion.

Finally, item (c) follows from the observation that, for every $F, K \in \mathcal{I}, E_{F}^{\lambda} \circ E_{K}^{\lambda} \subseteq E_{F K}^{\lambda}$.

Of course, if $M$ is an abelian unitary magma and $\mathcal{I}$ is a magmatic ideal, then $\mathcal{E}_{\mathcal{I}}^{\lambda}=\mathcal{E}_{\mathcal{I}}^{\rho}$. Note that, if $M$ is an abelian loop with the right inverse property, then it has the inverse property. In the next remark we discuss a situation in which the left and the right magmatic entourage structure are asymorphic, even though the may not be equal.

Remark 2.7. Let $G$ be a group and $\mathcal{I}$ be a magmatic ideal. Note that $\mathcal{I}^{-1}=\left\{K^{-1} \mid K \in \mathcal{I}\right\}$ is still a magmatic ideal and, more precisely, if $\mathcal{I}$ is a loop ideal or a monoid ideal, then so it is $\mathcal{I}^{-1}$. Consider the map $i: G \rightarrow G$ such that $i(g)=g^{-1}$, for every $g \in G$. Then $i:\left(G, \mathcal{E}_{\mathcal{I}}^{\lambda}\right) \rightarrow\left(G, \mathcal{E}_{\mathcal{I}^{-1}}^{\rho}\right)$ is an asymorphism. In fact, for every $K \in \mathcal{I}$ and every $(x, x k) \in E_{K}^{\lambda},(i \times i)(x, x k)=\left(x^{-1}, k^{-1} x^{-1}\right) \in E_{K^{-1}}^{\rho}$. The same conclusion holds if $\mathcal{I}$ is a loop ideal, a monoid ideal, or a group ideal. In particular, if $\mathcal{I}$ is a loop ideal or a group ideal, then $\mathcal{I}=\mathcal{I}^{-1}$ and thus $\left(G, \mathcal{E}_{\mathcal{I}}^{\lambda}\right)$ and $\left(G, \mathcal{E}_{\mathcal{I}}^{\rho}\right)$ are asymorphic. Hence, on a group $G$, if $\mathcal{I}$ is a loop ideal, we simply write $\mathcal{E}_{\mathcal{I}}$ instead of both $\mathcal{E}_{\mathcal{I}}^{\lambda}$ and $\mathcal{E}_{\mathcal{I}}^{\rho}$ when there is no risk of ambiguity.

A family of maps $\mathcal{F}$ between two entourage spaces $\left(X, \mathcal{E}_{X}\right)$ and $\left(Y, \mathcal{E}_{Y}\right)$ is equi-bornologous if, for every $E \in \mathcal{E}_{X}$, there exists $F \in \mathcal{E}_{Y}$ such that $(f \times f)(E) \subseteq F$, for every $f \in \mathcal{F}$.

If $M$ is an unitary magma and $\mathcal{E}$ is an entourage structure on it, we define the following families of maps, which are the left and the right shifts in $M$ :

$$
\mathcal{S}_{M}^{\lambda}=\left\{s_{x}^{\lambda} \mid x \in M\right\} \quad \text { and } \mathcal{S}_{M}^{\rho}=\left\{s_{x}^{\rho} \mid x \in M\right\} \text {, where, for every } x, y \in M, s_{x}^{\lambda}(y)=x y \text { and } s_{x}^{\rho}(y)=y x .
$$

Remark 2.8. (a) For every finitely generated monoid $M$, if $\Sigma$ is a finite generating set, then the quasi-coarse space $\left(M, \mathcal{E}_{d_{\Sigma}^{\lambda}}\right)$ makes the family $\mathcal{S}_{M}^{\lambda}=\left\{s_{x}^{\lambda} \mid x \in M\right\}$ equi-bornologous, since $d_{\Sigma}^{\lambda}$ is left-non-expanding.

(b) Let $M$ be a monoid and $\mathcal{I}$ be a monoid ideal on $M$. Then $\mathcal{S}_{M}^{\lambda}$ and $\mathcal{S}_{M}^{\rho}$ are equi-bornologous if $M$ is endowed with the left monoid quasi-coarse structure $\mathcal{E}_{\mathcal{I}}^{\lambda}$ and the right monoid quasi-coarse structure $\mathcal{E}_{\mathcal{I}}^{\rho}$, respectively. In fact, let $e \in K \in \mathcal{I}$. Then, for every $x \in M$ and every $(y, y k) \in E_{K}^{\lambda}$,

$$
\left(s_{x}^{\lambda} \times s_{x}^{\lambda}\right)(y, y k)=(x y, x y k)=x y(e, k) \in E_{K}^{\lambda} .
$$

(c) The shifts of the unitary magmas $(\mathbb{Z} \cup\{e\},-, e)$ and $(\mathbb{Q} \cup\{e\}, /, e)$ are equi-bornologous, once those magmas are equipped with the finitary magmatic entourage structure. Those result follow from a more general statement.

(d) Let $M$ be a unitary magma such that there exists a map $r: M \rightarrow M$ with the property that $a(b c)=(a b) r(c)$, for every $a, b, c \in M$. Hence, we claim that, for every magmatic ideal $\mathcal{I}$ on $M$ such that $r(\mathcal{I})=\{r(K) \mid K \in \mathcal{I}\} \subseteq \mathcal{I}$, the family of left shifts is equi-bornologous. Let $e \in K \in \mathcal{I}$ be a generic element of the group ideal. Then, for every $a, b \in M$,

$$
s_{a}^{\lambda}\left(B_{\mathcal{I}}(b, K)\right)=s_{a}^{\lambda}(b K)=a(b K)=(a b) r(K)=s_{a}^{\lambda}(b) r(K)=B_{\mathcal{I}}\left(s_{a}^{\lambda}(b), r(K)\right),
$$

which concludes the proof, since $r(K) \in \mathcal{I}$ and $e=r(e) \in r(K)$.

The next proposition shows the importance of having the families of lift (right) shifts, defined in (6), equi-bornologous. We state the result just for $\mathcal{S}_{M}^{\lambda}$, but similar conclusions hold also for $\mathcal{S}_{M}^{\rho}$.

Proposition 2.9. Let $M$ be an unitary magma and $\mathcal{E}$ be an entourage structure over $M$ such that $\mathcal{S}_{M}^{\lambda}$ is equi-bornologous. Let $\mathcal{I}=\{E[e] \mid E \in \mathcal{E}\}$. Then:

(a) $\mathcal{I}$ is a magmatic ideal and $\mathcal{E}_{\mathcal{I}}^{\lambda} \subseteq \mathcal{E}$;

(b) if $M$ is a loop with the inverse property and two-side inverses and $\mathcal{E}$ is a semi-coarse structure, then $\mathcal{I}$ is a loop ideal and $\mathcal{E}_{\mathcal{I}}^{\lambda}=\mathcal{E}$

(c) if $M$ is a monoid and $\mathcal{E}$ is a quasi-coarse structure, then $\mathcal{I}$ is a monoid ideal and $\mathcal{E}_{\mathcal{I}}^{\lambda} \subseteq \mathcal{E}$;

(d) if $M$ is a group and $\mathcal{E}$ is a coarse structure, then $\mathcal{I}$ is a group ideal and $\mathcal{E}_{\mathcal{I}}^{\lambda}=\mathcal{E}$.

Proof. The first assertion of item (a) is trivial since $E[e] \cup F[e]=(E \cup F)[e]$, for every $E, F \in \mathcal{E}$. Let now $F \in \mathcal{E}$ be an arbitrary entourage, and so $F[e]$ be an arbitrary element of $\mathcal{I}$. Since $\mathcal{S}_{M}^{\lambda}$ is equi-bornologous, there exists $F^{\prime} \in \mathcal{E}$ such that, for every $x \in M,\left(s_{x}^{\lambda} \times s_{x}^{\lambda}\right)(F) \subseteq E^{\prime}$. Then, for every $(x, y) \in E_{F[e]}^{\lambda}$, there exists $k \in F[e]$ such that $y=x k$. Then

$$
(x, y)=(x, x k)=\left(s_{x}^{\lambda} \times s_{x}^{\lambda}\right)(e, k) \in\left(s_{x}^{\lambda} \times s_{x}^{\lambda}\right)(F) \subseteq F^{\prime}
$$

and so $E_{F[e]}^{\lambda} \subseteq F^{\prime}$. Hence, $\mathcal{E}_{\mathcal{I}}^{\lambda} \subseteq \mathcal{E}$.

(b) Let $E \in \mathcal{E}$ and let $x$ be an arbitrary element of $E[e]$. Then,

$$
\left(e, x^{-1}\right)=\left(s_{x^{-1}}^{\lambda} \times s_{x^{-1}}^{\lambda}\right)(x, e) \in\left(s_{x^{-1}}^{\lambda} \times s_{x^{-1}}^{\lambda}\right)\left(E^{-1}\right) \subseteq F,
$$

where $F$ can be chosen independently from the choice of $x$ in $E[e]$, since $\mathcal{S}_{M}^{\lambda}$ is equi-bornologous. Thus $E[e]^{-1} \subseteq F[e] \in \mathcal{I}$. 
Consider now an arbitrary entourage $E \in \mathcal{E}$. We want to show that there exists $F \in \mathcal{E}$, such that $E \subseteq E_{F[e]}^{\lambda}$. Let $(x, y) \in E$ and denote by $F \in \mathcal{E}$ an entourage such that $\left(s_{z}^{\lambda} \times s_{z}^{\lambda}\right)(E) \subseteq F$, for every $z \in M$. Then

$$
(x, y)=\left(s_{x}^{\lambda} \times s_{x}^{\lambda}\right)\left(e, x^{-1} y\right), \quad \text { where }\left(e, x^{-1} y\right)=\left(s_{x^{-1}}^{\lambda} \times s_{x^{-1}}^{\lambda}\right)(x, y) \subseteq F,
$$

and thus $(x, y) \in E_{F[e]}$. Note that in (77) we used that $M$ has the inverse property and two-sided inverses.

(c) Thanks to item (a), we only need to show that $\mathcal{I}$ is a monoid ideal. Take $E, F \in \mathcal{E}$ and consider $E[e] \cdot F[e]$. Let $x \in E[e]$ and $y \in F[e]$ be two arbitrary elements, which means that $(e, x) \in E$ and $(e, y) \in F$. Denote by $E^{\prime} \in \mathcal{E}$ an entourage such that $\left(s_{x}^{\lambda} \times s_{x}^{\lambda}\right)(F) \subseteq E^{\prime}$, for every $x \in M$. Then

$$
(e, x y)=(e, x) \circ(x, x y) \in E \circ\left(s_{x}^{\lambda} \times s_{x}^{\lambda}\right)(F) \subseteq E \circ E^{\prime} \in \mathcal{E},
$$

which shows that $x y \in\left(E \circ E^{\prime}\right)[e]$, and thus $E[e] F[e] \subseteq\left(E \circ E^{\prime}\right)[e] \in \mathcal{I}$.

Finally, item (d) descends from items (b) and (c).

Remark 2.10. Let $M$ be a monoid generated by a finite subset $\Sigma$. By Remark 2.8 and Proposition 2.9] $\mathcal{E}_{\mathcal{I}}^{\lambda} \subseteq \mathcal{E}_{d_{\Sigma}^{\lambda}}$, where $\mathcal{I}$ is the family of all subsets of $\left(M, d_{\Sigma}^{\lambda}\right)$ bounded from e, i.e., contained in some ball centred at $e$. More precisely, $\mathcal{I}=[M]^{<\infty}$. We claim that $\mathcal{E}_{\mathcal{I}}^{\lambda}=\mathcal{E}_{d_{\Sigma}^{\lambda}}$. Let $R \geq 0$ and define $F_{R}=\left\{\sigma_{1} \cdots \sigma_{n} \mid n \leq R, \sigma_{i} \in \Sigma, \forall i=1, \ldots, m\right\}$. Then $F_{R} \in \mathcal{I}$. Moreover, if $d_{\Sigma}^{\lambda}(x, y) \leq R$, then $y \in x F_{R}$. Hence $E_{R} \subseteq E_{F_{R}}^{\lambda}$, which shows the desired equality. Similarly, $\mathcal{E}_{\mathcal{I}}^{\rho}=\mathcal{E}_{d_{\Sigma}^{\rho}}$

Let $f: M \rightarrow N$ be a map between two unitary magmas. Then $f$ is called a homomorphism if, for every $g, h \in M$, $f(g h)=f(g) f(h)$ and $f\left(e_{M}\right)=e_{N}$. Propositions 2.11 and 2.13 are relaxed versions of classical results in the framework of coarse structures on groups $([14)$.

Proposition 2.11. Let $f: M \rightarrow N$ be a homomorphism between unitary magmas, and $\mathcal{I}_{M}$ and $\mathcal{I}_{N}$ be two magmatic ideals on $M$ and $N$, respectively. Then the following properties are equivalent:

(a) $f\left(\mathcal{I}_{M}\right)=\{f(K) \mid K \in \mathcal{I}\} \subseteq \mathcal{I}_{N}$;

(b) $f:\left(M, \mathcal{E}_{\mathcal{I}_{M}}^{\lambda}\right) \rightarrow\left(N, \mathcal{E}_{\mathcal{I}_{N}}^{\lambda}\right)$ is bornologous;

(c) $f:\left(M, \mathcal{E}_{\mathcal{I}_{M}}^{\rho^{M}}\right) \rightarrow\left(N, \mathcal{E}_{\mathcal{I}_{N}}^{\rho^{N}}\right)$ is bornologous.

Proof. The implications (b) $\rightarrow$ (a) and (c) $\rightarrow$ (a) are trivial. In fact, for every $K \in \mathcal{I}_{M}, f(K)=f\left(E_{K}^{\lambda}[e]\right) \subseteq\left((f \times f)\left(E_{K}^{\lambda}\right)\right)[e]$. Let us now prove $(\mathrm{a}) \rightarrow(\mathrm{b})$, and $(\mathrm{a}) \rightarrow(\mathrm{c})$ can be similarly shown. Let $K \in \mathcal{I}$. Then, for every $(x, x k) \in E_{K}^{\lambda},(f \times f)(x, x k)=$ $(f(x), f(x) f(k)) \in E_{f(K)}^{\lambda}$, and thus $(f \times f)\left(E_{K}^{\lambda}\right) \subseteq E_{f(K)}^{\lambda} \in \mathcal{E}_{\mathcal{I}_{N}}^{\lambda}$.

Fact 2.12. Let $M$ and $N$ be two loops and $f: M \rightarrow N$ be a homomorphism between them.

(a) $f(x)^{\lambda}=f\left(x^{\lambda}\right)$ and $f(x)^{\rho}=f\left(x^{\rho}\right)$, for every $x \in M$.

(b) $f(M)$ is a subloop of $N$.

(c) If $M$ has two-sided inverses, then $f(N)$ has also two-sided inverses.

Proof. (a) Let $x \in M$. Then

$$
f(x)^{\lambda} f(x)=e=f(e)=f\left(x^{\lambda} x\right)=f\left(x^{\lambda}\right) f(x) \quad \text { and } \quad f(x) f(x)^{\rho}=e=f(e)=f\left(x x^{\rho}\right)=f(x) f\left(x^{\rho}\right),
$$

and so the conclusion follows by uniqueness of the solutions of (5).

(b) Let $f(a)$ and $f(b)$ be two elements in $f(M)$. Then there exists a unique $x \in M$ such that $a x=b$ and so $f(a)=f(x) f(b)$. Moreover, the solution $f(x)$ is unique since $N$ is a loop.

(c) For every $f(x) \in N, f(x)^{\lambda}=f\left(x^{\lambda}\right)=f\left(x^{\rho}\right)=f(x)^{\rho}$.

Proposition 2.13. Let $f: M \rightarrow N$ be a homomorphism between two loops with inverse properties, and $\mathcal{I}_{M}$ and $\mathcal{I}_{N}$ be two magmatic ideals on $M$ and $N$, respectively. Assume that $M$ has two-side inverses and $f$ is a homomorphism. Then the following properties are equivalent:

(a) $f^{-1}\left(\mathcal{I}_{N}\right) \subseteq \mathcal{I}_{M}$;

(b) $f:\left(M, \mathcal{E}_{\mathcal{I}_{M}}^{\lambda}\right) \rightarrow\left(N, \mathcal{E}_{\mathcal{I}_{N}}^{\lambda}\right)$ is effectively proper.

Proof. Implication (b) $\rightarrow$ (a) is trivial. In fact, for every $K \in \mathcal{I}_{N}, f^{-1}(K)=f^{-1}\left(E_{K}^{\lambda}[e]\right) \subseteq\left((f \times f)^{-1}\left(E_{K}^{\lambda}\right)\right)[e]$ and $(f \times f)^{-1}\left(E_{K}^{\lambda}\right) \in \mathcal{I}_{M}$. Conversely, let $e \in K \in \mathcal{I}_{N}$ and $(x, y) \in(f \times f)^{-1}\left(E_{K}^{\lambda}\right)$. Then $f(y) \in f(x) K$, which implies that $f\left(x^{-1} y\right) \in K$. Hence, $x^{-1} y \in f^{-1}(K)$ and thus $(x, y) \in E_{f^{-1}(K)}^{\lambda}$.

\section{Categories of entourage spaces}

A concrete category $(\mathcal{X}, \mathrm{U})$ is a pair where $\mathcal{X}$ is a category and $\mathrm{U}: \mathcal{X} \rightarrow$ Set is a faithful functor (i.e., such that, for every $f, g \in \operatorname{Mor}_{\mathcal{X}}(X, Y), \mathrm{U} f=\mathrm{U} g$ if and only if $f=g$ ). In that situation, the fiber of a set $A$ is the family of all $X$ in $\mathcal{X}$ such that $\mathrm{U} X=A$. If $(\mathcal{X}, \mathrm{U})$ is a concrete category and $X$ and $Y$ are two objects of $\mathcal{X}$, a morphism $f: \mathrm{U} X \rightarrow \mathrm{U} Y$ is a $\mathcal{X}$-morphism with respect to $X$ and $Y$ whenever there exists $\bar{f} \in \operatorname{Mor}_{\mathcal{X}}(X, Y)$ such that $\mathrm{U} \bar{f}=f$. 
A source in a category $\mathcal{X}$ is a family (possibly a proper class) $\left\{f_{i}: X \rightarrow X_{i}\right\}_{i \in I}$ of morphisms of $\mathcal{X}$. Its dual notion is the one of sink. A sink in a category $\mathcal{X}$ is a family $\left\{f_{i}: X_{i} \rightarrow X\right\}_{i \in I}$ of morphisms of $\mathcal{X}$.

Suppose now that $(\mathcal{X}, \mathrm{U})$ is a concrete category. A source $\left\{f_{i}: X \rightarrow X_{i}\right\}_{i \in I}$ of $\mathcal{X}$ is initial if, for every morphism $f: \mathrm{U} A \rightarrow \mathrm{U} X$ of Set, such that $\mathrm{U} f_{i} \circ \mathrm{U} f: \mathrm{U} A \rightarrow \mathrm{U} X_{i}$ is an $\mathcal{X}$-morphism, then $f$ is an $\mathcal{X}$-morphism. An initial lifting of a source $\left\{f_{i}: A \rightarrow \mathrm{U} X_{i}\right\}$ in Set, where, for every $i \in I, X_{i}$ is an object of $\mathcal{X}$, is an initial source $\left\{g_{i}: B \rightarrow X_{i}\right\}_{i \in I}$ of $\mathcal{X}$ such that $\mathrm{U} B=A$ and $\mathrm{U} g_{i}=f_{i}$, for every $i \in I$.

Definition $3.1([2])$. A concrete category $(\mathcal{X}, \mathrm{U})$ is topological if:

(a) $\mathrm{U}$ is amnestic (i.e. $f=1_{X}$, whenever $f: X \rightarrow X$ is an isomorphism of $\mathcal{X}$ such that $\mathrm{U} f=1_{\mathrm{U} X}$ );

(b) $\mathrm{U}$ is transportable (i.e., for every object $A$ of $\mathcal{X}$ and every isomorphism $h: \mathrm{U} A \rightarrow X$ of Set, there exists an object $B$ of $\mathcal{X}$ and an isomorphism $f: A \rightarrow B$ of $\mathcal{X}$ such that $\mathrm{U} f=h$ );

(c) constant maps are morphisms of $\mathcal{X}$;

(d) U has small fibers (i.e., the fibers are sets);

(e) every singleton of Set has a unique element in its fiber;

(f) every source $\left\{f_{i}: A \rightarrow U X_{i}\right\}_{i \in I}$ of Set, has an initial lifting.

Let us define the concrete category Entou, whose objects are entourage spaces and whose morphisms are bornologous maps between them. Moreover, we consider three full subcategories of Entou, namely, SCoarse, whose objects are semicoarse spaces, QCoarse, the subcategory of quasi-coarse spaces, and, finally, Coarse, the subcategory of coarse spaces. All of those categories $\mathcal{X}$ are concrete and so they have forgetful functors (i.e., faithful functors) $\mathrm{U}_{\mathcal{X}}: \mathcal{X} \rightarrow$ Set. Moreover, there are the following forgetful functors:

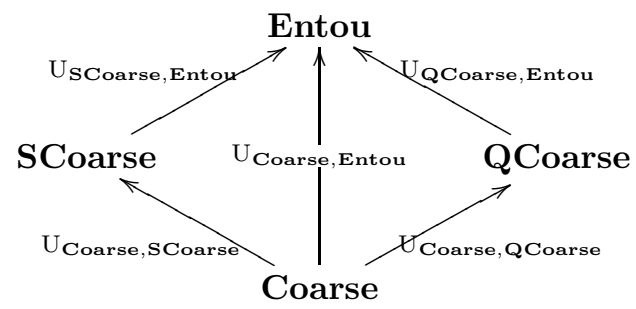

We write $\mathrm{U}$ instead of both $\mathrm{U}_{\mathcal{X}, \mathcal{Y}}: \mathcal{X} \rightarrow \mathcal{Y}$ and $\mathrm{U}_{\mathcal{X}}: \mathcal{X} \rightarrow$ Set if there is no risk of ambiguity.

Theorem 3.2. The categories Entou, SCoarse, QCoarse and Coarse are topological.

The only thing we need to verify is that those categories allow for lifting of initial sources since the other requests can be easily checked. Let $f: X \rightarrow(Y, \mathcal{E})$ be a map between a set and an entourage space. We define the initial entourage structure $f_{*} \mathcal{E}$ as the entourage structure over $X$ generated by the base $\left\{(f \times f)^{-1}(E) \mid E \in \mathcal{E}\right\}$. If $\mathcal{E}$ is a semi-coarse structure (quasi-coarse structure), then $f_{*} \mathcal{E}$ is a semi-coarse structure (quasi-coarse structure, respectively). Moreover, $f:\left(X, f_{*} \mathcal{E}\right) \rightarrow(Y, \mathcal{E})$ is bornologous and effectively proper.

Proof of Theorem 3.2. Let $\left\{f_{i}: X \rightarrow\left(Y_{i}, \mathcal{E}_{i}\right)\right\}_{i \in I}$ be a source of maps from a set to a family of entourage spaces. Define the entourage structure $\mathcal{E}$ over $X$ as $\mathcal{E}=\bigcap_{i \in I}\left(f_{i}\right)_{*} \mathcal{E}_{i}$. If $\mathcal{E}_{i}$ is a semi-coarse structure (a quasi-coarse structure), for every $i \in I$, then $\mathcal{E}$ is a semi-coarse structure (a quasi-coarse structure, respectively).

A morphism $\alpha: X \rightarrow X^{\prime}$, in a category $\mathcal{X}$, is called:

- an epimorphism if every pair of morphisms $\beta, \gamma: X^{\prime} \rightarrow X^{\prime \prime}$ such that $\beta \circ \alpha=\gamma \circ \alpha$ satisfies $\beta=\gamma$;

- a monomorphism if every pair of morphisms $\beta, \gamma: X^{\prime \prime} \rightarrow X$ such that $\alpha \circ \beta=\alpha \circ \gamma$ satisfies $\beta=\gamma$;

- a bimorphism if it is both epimorphism and monomorphism.

Thanks to Theorem 3.2 the fact that in those categories the epimorphisms are surjective morphisms and the monomorphisms are injective morphisms follows ([5]). In particular those four categories are not balanced. Recall that a category $\mathcal{X}$ is balanced if every bimorphism is an isomorphism. The fact that Coarse is topological and it is not balanced was already proved in [6].

A category $\mathcal{X}$ is cowellpowered if for every object $X$ and every source $\left\{e_{i}: X \rightarrow X_{i}\right\}_{i \in I}$ of epimorphisms (possibly a proper class), there exists a set $\left\{e_{j}\right\}_{j \in J}, J \subseteq I$, such that, for every $i \in I$, there exists $j \in J$ and an isomorphism $f$ of $\mathcal{X}$ such that $e_{i}=f \circ e_{j}$.

Since the epimorphisms of Entou, SCoarse, QCoarse, and Coarse are surjective morphisms, those categories are cowellpowered. Moreover, in 23] it was proved that every epireflective subcategory of Coarse is cowellpowered. Hence the following question naturally arises.

Question 3.3. Does there exist a subcategory of Entou containing Coarse which is not cowellpowered?

\subsection{Functors between the four categories Entou, SCoarse, QCoarse, and Coarse}

We want to study the relationships between Entou, SCoarse, QCoarse, and Coarse, and, in order to do that, we define some useful functors between the four categories, which will be summarised in the diagram (9). All these functors 
will only be defined on the objects, since the morphisms are "fixed" (i.e., if $\mathrm{F}: \mathcal{X} \rightarrow \mathcal{Y}$ is one of the functors that we are going to define and $f: X \rightarrow Y$ is a morphism of $\mathcal{X}$, then $\mathrm{U}(\mathrm{F} f)=\mathrm{U} f$ ).

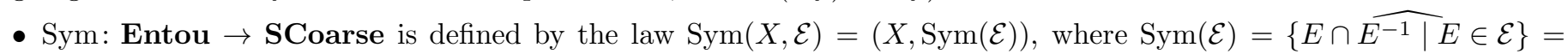
$\mathcal{E} \cap \mathcal{E}^{-1}$, for every $(X, \mathcal{E}) \in$ Entou. In a similar way, Sym: QCoarse $\rightarrow$ Coarse is defined.

- USym: Entou $\rightarrow$ SCoarse is defined by the law $\operatorname{USym}(X, \mathcal{E})=(X, \operatorname{USym}(\mathcal{E}))$, where $\operatorname{USym}(\mathcal{E})=\left\{E \cup \widehat{E^{-1} \mid} E \in \mathcal{E}\right\}=$ $\widehat{\mathcal{E} \cup \mathcal{E}^{-1}}$, for every $(X, \mathcal{E}) \in$ Entou.

- $\mathrm{W}:$ Entou $\rightarrow$ QCoarse is defined by the law $\mathrm{W}(X, \mathcal{E})=(X, \mathrm{~W}(\mathcal{E}))$, for every $(X, \mathcal{E}) \in$ Entou, where $\mathrm{W}(\mathcal{E})=$ $\left\{E^{n} \mid n \widehat{\in \mathbb{N}}, E \in \mathcal{E}\right\}$ and, for every $E \in \mathcal{E}$

$$
E^{n}=\underbrace{E \circ \cdots \circ E}_{n \text { times }} \text {. }
$$

Similarly, W : SCoarse $\rightarrow$ Coarse is defined.

If we also consider the composite functor WoUSym $\circ \mathrm{U}_{\mathbf{Q C o a r s e} \text {,Entou }}$ : QCoarse $\rightarrow$ Coarse, the situation can be represented by the following diagram:

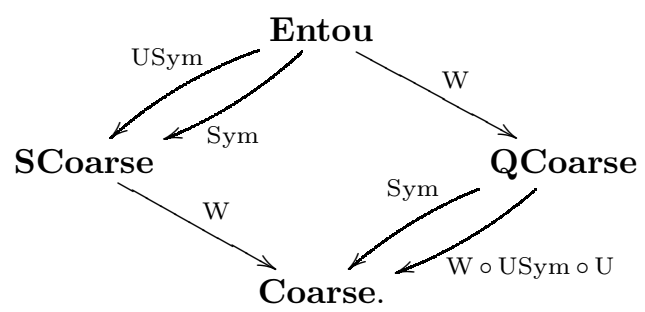

There is another endofunctor $\mathrm{J}$ of Entou that it is worth mentioning. Every entourage space $(X, \mathcal{E})$ is associated to $\mathrm{J}(X, \mathcal{E})=\left(X, \mathcal{E}^{-1}\right)$ and every morphism $f \in \operatorname{Mor}_{\mathbf{E n t o u}}(X, Y)$ is fixed, i.e., $\mathrm{U} f=\mathrm{U}(\mathrm{J} f)$. Since, for every entourage $E$ of $X,(f \times f)\left(E^{-1}\right)=((f \times f)(E))^{-1}, \mathrm{~J} f$ is bornologous whenever $f$ is bornologous, and so $\mathrm{J}$ is a functor. Note that $\left.\mathrm{J}\right|_{\text {SCoarse }}$ is the identity functor of SCoarse.

Remark 3.4. (a) Note that the functor Sym generalises the definition of the semi-coarse hyperstructure from the entourage hyperstructure (see 2.3 for the definitions). More precisely, if $(X, \mathcal{E})$ is an entourage space, then $\operatorname{Sym}(\mathcal{P}(X), \mathcal{H}(\mathcal{E}))=(\mathcal{P}(X), \exp \mathcal{E})$.

(b) It is not true in general that, if $X$ is a quasi-coarse space, $\exp X=\exp (\operatorname{Sym} X)$. In fact, let $X=\mathbb{Z}$ with the relation entourage structure induced by the usual order relation, which is a quasi-coarse structure. Then $\operatorname{Sym} X$ is $X$ endowed with the discrete coarse structure. However, $2 \mathbb{Z}$ and $2 \mathbb{Z}+1$ belong to the same connected component of $\exp X$.

In the following example we provide a semi-coarse structure that admits no maximal coarse structure that is finer than the original one.

Example 3.5. Consider the following extended semi-metric $d$ on $\mathbb{Z}^{2}$ : for every $(x, y),(z, w) \in \mathbb{Z}^{2}$,

$$
d((x, y),(z, w))=\left\{\begin{array}{lr}
|x-z| & \text { if } y=w \\
|y-w| & \text { if } x=z \\
\infty & \text { otherwise }
\end{array}\right.
$$

Then $\left(\mathbb{Z}^{2}, \mathcal{E}_{d}\right)$ is a semi-coarse space. We claim that there are two different maximal coarse-structures $\mathcal{E}_{1}$ and $\mathcal{E}_{2}$ on $\mathbb{Z}^{2}$ which are finer then $\mathcal{E}_{d}$.

Define the following extended metrics $d_{1}$ and $d_{2}$ as follows: for every $(x, y),(z, w) \in \mathbb{Z}^{2}$,

$$
d_{1}((x, y),(z, w))=\left\{\begin{array}{ll}
|x-z| & \text { if } y=w, \\
\infty & \text { otherwise. }
\end{array} \quad d_{2}((x, y),(z, w))= \begin{cases}|y-w| & \text { if } x=z, \\
\infty & \text { otherwise }\end{cases}\right.
$$

Then $\mathcal{E}_{1}=\mathcal{E}_{d_{1}}$ and $\mathcal{E}_{2}=\mathcal{E}_{d_{2}}$ satisfy the desired properties.

Let us now recall some other basic categorical definitions from [1]. Let $\mathrm{F}, \mathrm{G}: \mathcal{X} \rightarrow \mathcal{Y}$ be two functors between two categories. A natural transformation $\eta$ from $F$ to $G$ (in symbols, $F \stackrel{\eta}{\rightarrow} G$ ) is a function that assign to each object $X$ of $\mathcal{X}$, a morphism $\eta_{X}: \mathrm{F} X \rightarrow \mathrm{G} X$ of $\mathcal{Y}$ such that, for every other morphism $f: X \rightarrow X^{\prime}$ of $\mathcal{X}$, the following diagram commutes:

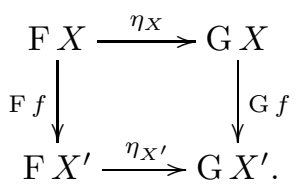

Let now $\mathrm{G}: \mathcal{X} \rightarrow \mathcal{Y}$ and $\mathrm{F}: \mathcal{Y} \rightarrow \mathcal{X}$ be two functors. Then $\mathrm{F}$ is co-adjoint for $\mathrm{G}$ (or $\mathrm{F}$ is left adjoint of $\mathrm{G}$, or $\mathrm{F}$ has a right adjoint $\mathrm{G}$ ) and $\mathrm{G}$ is adjoint for $\mathrm{F}$ (or $\mathrm{G}$ is right adjoint of $\mathrm{F}$, or $\mathrm{G}$ has a left adjoint $\mathrm{F}$ ) if there exist two natural 
transformations $\eta: i d \mathcal{Y} \rightarrow \mathrm{G} \circ \mathrm{F}$ (called unit) and $\varepsilon: \mathrm{F} \circ \mathrm{G} \rightarrow i d_{\mathcal{X}}$ (called co-unit) such that the following two triangular identities hold: for every object $X \in \mathcal{X}$ and every $Y \in \mathcal{Y}$, the following triangles commutes:

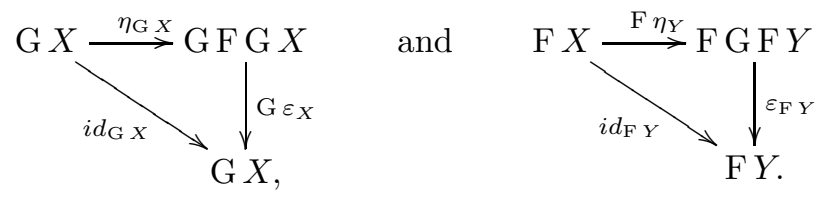

Let $\mathcal{Y}$ be a full subcategory of a category $\mathcal{X}$. Then $\mathcal{Y}$ is reflective in $\mathcal{X}$ if there exists a functor $\mathrm{G}: \mathcal{X} \rightarrow \mathcal{Y}$, called reflector, which is a co-adjoint for the inclusion functor $\mathrm{I}: \mathcal{Y} \rightarrow \mathcal{X}$. Dually, $\mathcal{Y}$ is coreflective in $\mathcal{X}$ if there exists a functor $\mathrm{G}: \mathcal{X} \rightarrow \mathcal{Y}$, called co-reflector, which is an adjoint for $\mathrm{I}: \mathcal{Y} \rightarrow \mathcal{X}$.

The functors previously defined can be used to prove the following theorem.

Theorem 3.6. (a) QCoarse is a reflective subcategory in Entou;

(b) SCoarse is a reflective and co-reflective subcategory in Entou;

(c) Coarse is a reflective subcategory in SCoarse;

(d) Coarse is a reflective and co-reflective subcategory in QCoarse.

Proof. The thesis follows by proving the following assertions:

- W is a reflector of I: QCoarse $\rightarrow$ Entou;

- USym is a reflector and Sym is a co-reflector of I: SCoarse $\rightarrow$ Entou;

- W is a reflector of I: Coarse $\rightarrow$ SCoarse;

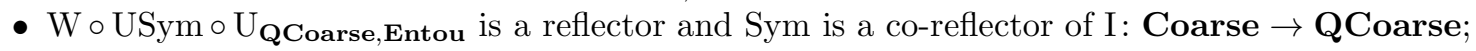

which are easy checks of the definitions.

Embeddings of reflective subcategories preserve limits (e.g., products), while embeddings of co-reflective subcategories preserve colimits (e.g., coproducts and quotients). See 1 for details. In 3.2, we prove that neither QCoarse is a coreflective subcategory in Entou, nor Coarse is a co-reflective subcategory in SCoarse since they do not preserve some colimits.

\subsection{Product, coproducts and quotients}

Let $\mathcal{X}$ be a category and $\left\{X_{i}\right\}_{i \in I}$ be a family of objects of $\mathcal{X}$. A source $\left\{p_{i}: X \rightarrow X_{i}\right\}_{i \in I}$, where $X$ is an object of $\mathcal{X}$, is the product of $\left\{X_{i}\right\}_{i \in I}$ in $\mathcal{X}$ if it satisfies the following universal property: for every other source $\left\{f_{i}: Y \rightarrow X_{i}\right\}_{i \in I}$, where $Y$ is another object of $\mathcal{X}$, there exists a unique morphism $f: Y \rightarrow X$ such that $f_{i}=p_{i} \circ f$, for every $i \in I$.

Let $\left\{\left(X_{i}, \mathcal{E}_{i}\right)\right\}_{i \in I}$ be a family of entourage spaces. Let $X=\Pi_{i} X_{i}$ and $p_{i}: X \rightarrow X_{i}$, for every $i \in I$ be the projection maps. Then the product entourage structure $\mathcal{E}=\Pi_{i} \mathcal{E}_{i}$ is defined as

$$
\mathcal{E}=\left\{\bigcap_{i \in I}\left(p_{i} \times p_{i}\right)^{-1}\left(\overline{\left.E_{i}\right) \mid} E_{i} \in \mathcal{E}_{i}, \forall i \in I\right\} .\right.
$$

We can check that $(X, \mathcal{E})$ is the product in Entou. As we have already pointed out, since Coarse is reflective in both SCoarse and QCoarse, which are reflective in Entou, these categories are stable under taking products. Hence, the same construction leads to the product in SCoarse, QCoarse and Coarse. The products in Coarse are well-known objects (see, for instance, [20, 6]).

Let $\mathcal{X}$ be a category and $\left\{X_{k}\right\}_{k \in I}$ be a family of objects of $\mathcal{X}$. A $\operatorname{sink}\left\{i_{k}: X_{k} \rightarrow X\right\}_{k \in I}$, where $X$ is an object of $\mathcal{X}$, is the coproduct of $\left\{X_{k}\right\}_{k \in I}$ in $\mathcal{X}$ if it satisfies the following universal property: for every other sink $\left\{f_{k}: X_{k} \rightarrow Y\right\}_{k \in I}$, where $Y$ is another object of $\mathcal{X}$, there exists a unique morphism $f: X \rightarrow Y$ such that $f_{k}=f \circ i_{k}$, for every $k \in I$.

Let $\left\{\left(X_{k}, \mathcal{E}_{k}\right)\right\}_{k \in I}$ be a family of entourage spaces. On the disjoint union $X=\bigsqcup_{k} X_{k}$ of the supports, we define the coproduct entourage structure $\mathcal{E}=\bigoplus_{k} \mathcal{E}_{k}$ as follows:

$$
\begin{gathered}
\mathcal{E}=\left\{E_{J, \varphi} \mid J \in[I]<\infty, \varphi: J \rightarrow \bigcup_{k \in I} \mathcal{E}_{k}, \varphi(k) \in \mathcal{E}_{k}, \forall k \in I\right\} \\
\text { and, for every such a } J \text { and } \varphi, \quad E_{J, \varphi}=\Delta_{X} \cup\left(\bigcup_{j \in J}\left(i_{j} \times i_{j}\right)(\varphi(j))\right) .
\end{gathered}
$$

It is not hard to check that $(X, \mathcal{E})$ is actually the coproduct in Entou, SCoarse, QCoarse and Coarse. However, note that we could not have concluded as in the product case that, once we proved that it is the coproduct of Entou, then it would automatically be the coproduct of the other categories. In fact, QCoarse is not coreflective in Entou and Coarse is not coreflective in SCoarse.

Let $(\mathcal{X}, \mathrm{U})$ be a concrete category. Let $X$ be an object of $\mathcal{X}, A$ be a set, and $f: \mathrm{U} X \rightarrow A$ be an epimorphism in Set (i.e., a surjective map). Then the quotient of $f$ and $X$ is a morphism $\bar{f}: X \rightarrow Y$ of $\mathcal{X}$ with $\mathrm{U} \bar{f}=f$, and that satisfies 
the following universal property: for every other morphism $g: \mathrm{U} Y \rightarrow \mathrm{U} Z$ of Set, $g$ is an $\mathcal{X}$-morphism, provided that $g \circ f$ is an $\mathcal{X}$-morphism.

We want to construct quotients in the four categories we are considering. Let $q:(X, \mathcal{E}) \rightarrow Y$ be a surjective map from an entourage space to a set. Then the quotient entourage structure on $Y$ is $q(\mathcal{E})=\{(q \times q)(E) \mid E \in \mathcal{E}\}$. Moreover, if $\mathcal{E}$ is a semi-coarse structure, then $(Y, q(\mathcal{E}))$ is a semi-coarse space and thus it is also the quotient structure in $\mathbf{S C o a r s e}$ However, as proved in [6], if $\mathcal{E}$ is a coarse structure, then $q(\mathcal{E})$ is not a quasi-coarse structure in general. Then the quotient structure in QCoarse (in Coarse) is $\overline{\mathcal{E}}^{q}$, where $\overline{\mathcal{E}}^{q}$ is the finest quasi-coarse structure (coarse structure, respectively) which contains $q(\mathcal{E})$, namely $\mathrm{W}((Y, q(\mathcal{E})))$. Hence, in particular, QCoarse is not co-reflective in Entou and Coarse is not co-reflective in SCoarse.

Moreover, [6, Theorem 4.12] can be modified in this setting. Recall that a surjective map $q:(X, \mathcal{E}) \rightarrow Y$ from a quasi-coarse space to a set is weakly soft ([6]) if, for every $E \in \mathcal{E}$, there exists $F \in \mathcal{E}$ such that $E \circ R_{q} \circ E \subseteq R_{q} \circ F \circ R_{q}$.

Theorem 3.7. Let $q:(X, \mathcal{E}) \rightarrow Y$ be a surjective map from a quasi-coarse space to a set. Then $q(\mathcal{E})$ is a quasi-coarse structure, and thus $\overline{\mathcal{E}}^{q}=q(\mathcal{E})$, if and only if $q$ is weakly soft. In particular, $q(\mathcal{E})$ is the categorical quotient structure of QCoarse (of Coarse, provided that $\mathcal{E}$ is a coarse structure) if and only if $q$ is weakly soft.

Proof. The proof that shows [6, Theorem 4.12] can be easily adapted to obtain this result.

\section{Equivalence relations induced by functors}

A very important notion in coarse geometry is the one of coarse equivalence $([21])$. Let $f, g: X \rightarrow(Y, \mathcal{E})$ be two maps from a set to a coarse space. Then $f$ and $g$ are close, and we denote this fact by $f \sim g$, if $\{(f(x), g(x)) \mid x \in X\} \in \mathcal{E}$. Since $\mathcal{E}$ is a coarse space, then $\sim$ is an equivalence relation. A subset $Y$ of a coarse space $(X, \mathcal{E})$ is large if there exists $E \in \mathcal{E}$ such that $E[Y]=\bigcup_{y \in Y} E[y]=X$. A map $f:\left(X, \mathcal{E}_{X}\right) \rightarrow\left(Y, \mathcal{E}_{Y}\right)$ between coarse spaces is a coarse equivalence if it is bornologous and there exists another bornologous map $g: Y \rightarrow X$ such that $g \circ f \sim i d_{X}$ and $f \circ g \sim i d_{Y}$.

Suppose that $\mathrm{F}$ is a functor from a category $\mathcal{X}$ to Coarse. Then a notion of closeness can be inherited by $\mathcal{X}$ from Coarse. Let $f, g: X \rightarrow Y$ be two morphisms of $\mathcal{X}$. We say that $f$ is F-close to $g$ (and we write $f \sim_{\mathrm{F}} g$ ) if $\mathrm{F} f$ is close to $\mathrm{F} g$ in Coarse. These new relations are equivalences. Moreover, a morphism $k: W \rightarrow Z$ of $\mathcal{X}$ is a $\mathrm{F}$-coarse inverse of a morphism $h: Z \rightarrow W$ if $k \circ h \sim_{\mathrm{F}} i d_{Z}$ and $h \circ k \sim_{\mathrm{F}} i d_{W}$. Thanks to this notion, we can define equivalences between the objects of Entou, SCoarse and QCoarse.

Definition 4.1. Suppose that $\mathcal{X}$ is a subcategory of Entou and $\mathrm{F}$ is a functor from $\mathcal{X}$ to Coarse. A map $f:\left(X, \mathcal{E}_{X}\right) \rightarrow$ $\left(Y, \mathcal{E}_{Y}\right)$ between two objects of $\mathcal{X}$ is a $\mathrm{F}$-coarse equivalence if $f:\left(X, \mathcal{E}_{X}\right) \rightarrow\left(Y, \mathcal{E}_{Y}\right)$ is bornologous and it has a bornologous F-coarse inverse $g:\left(Y, \mathcal{E}_{Y}\right) \rightarrow\left(X, \mathcal{E}_{X}\right)$. In this case, $\left(X, \mathcal{E}_{X}\right)$ and $\left(Y, \mathcal{E}_{Y}\right)$ are called F-coarsely equivalent.

The concept just introduced induces an equivalence relation between objects of $\mathcal{X}$.

In particular, in this section we focus our attention to the equivalence induced by the functor Sym: QCoarse $\rightarrow$ Coarse. In Theorem 4.4 we characterise Sym-coarse equivalences.

A map $f:\left(X, \mathcal{E}_{X}\right) \rightarrow\left(Y, \mathcal{E}_{Y}\right)$ between quasi-coarse spaces is large-scale surjective if $f(X)$ is large in $\operatorname{Sym}\left(Y, \mathcal{E}_{Y}\right)$. If $f$ is also large-scale injective, then it is large-scale bijective. The following proposition characterises large-scale bijective maps between quasi-coarse spaces.

Proposition 4.2. Let $f:\left(X, \mathcal{E}_{X}\right) \rightarrow\left(Y, \mathcal{E}_{Y}\right)$ be a map between quasi-coarse space. Then $f$ is large-scale bijective if and only if it has a Sym-coarse inverse. In particular, every Sym-coarse inverse is large-scale bijective.

Proof. $(\rightarrow)$ Let $M=M^{-1} \in \operatorname{Sym}\left(\mathcal{E}_{Y}\right) \subseteq \mathcal{E}_{Y}$ be an entourage such that $M[f(X)]=Y$. For every $y \in Y$, there exists $x_{y} \in X$ such that $\left(y, f\left(x_{y}\right)\right) \in M$. If $y \in f(X)$, suppose that $x_{y} \in f^{-1}(y)$. Define $g: Y \rightarrow X$ with the following law: $g(y)=x_{y}$, for every $y \in Y$. Then $(f(g(y)), y) \in M$ for every $y \in Y$, which witnesses that $f \circ g \sim_{\text {Sym }} i d_{Y}$. The fact that $f$ is large-scale injective proves that $g \circ f \sim_{\text {Sym }} i d_{X}$.

$(\leftarrow)$ Let now $g: Y \rightarrow X$ be a Sym-coarse inverse of $f$. Let $M=M^{-1} \in \mathcal{E}_{X}$ and $N=N^{-1} \in \mathcal{E}_{Y}$ be two entourages that demonstrate that $g \circ f \sim_{\mathrm{Sym}} i d_{X}$ and $f \circ g \sim_{\mathrm{Sym}} i d_{Y}$, respectively. Note that, for every $y \in Y, f(g(y)) \in f(X)$ and $(y, f(g(y))),(f(g(y)), y) \in N$. Hence $f$ is large-scale surjective. Moreover, since $R_{f} \subseteq M \circ M, f$ is large-scale injective.

The last assertion is trivial since, if $g$ is a Sym-coarse inverse of $f$, then $f$ is a Sym-coarse inverse of $g$.

Proposition 4.3. Let $f:\left(X, \mathcal{E}_{X}\right) \rightarrow\left(Y, \mathcal{E}_{Y}\right)$ be a large-scale bijective map between quasi-coarse spaces and let $g$ be a Sym-coarse inverse of $f$. Then, the following properties are equivalent:

(a) $f$ is bornologous;

(b) $g$ is weakly uniformly bounded copreserving;

(c) $g$ is uniformly bounded copreserving;

(d) $g$ is effectively proper.

Moreover, every other Sym-coarse inverse $h$ of $g$ satisfies $h \sim_{\text {Sym }} f$. 
Proof. Since $g$ is large-scale injective, the equivalences (b) $\leftrightarrow(\mathrm{c}) \leftrightarrow(\mathrm{d})$ descend from Proposition 1.7. Suppose now that $f$ is bornologous. Let $E \in \mathcal{E}_{X}$ and consider $(g \times g)^{-1}(E)$. Denote by $M=M^{-1}$ the entourage of $\mathcal{E}_{Y}$ such that $(f(g(z)), z) \in M$, for every $z \in Y$. Then, for every $(x, y) \in(g \times g)^{-1}(E)$,

$$
(x, y)=(x, f(g(x))) \circ(f(g(x)), f(g(y))) \circ(f(g(y)), y) \in M \circ(f \times f)(E) \circ M \in \mathcal{E}_{Y} .
$$

Conversely, suppose that $g$ is effectively proper. Denote by $N=N^{-1} \in \mathcal{E}_{X}$ the entourage which witnesses that $g \circ f \sim_{\mathrm{Sym}} i d_{X}$. Let $E \in \mathcal{E}_{X}$ and $(x, y) \in E$. Then

$$
(g(f(x)), g(f(y)))=(g(f(x)), x) \circ(x, y) \circ(y, g(f(y))) \in N \circ E \circ N \in \mathcal{E}_{X}
$$

and thus $(f(x), f(y)) \in(g \times g)^{-1}(N \circ E \circ N) \in \mathcal{E}_{Y}$.

Finally, if $h$ is another Sym-coarse inverse of $g$, then, for every $x \in X,(g(f(x)), g(h(x)))=(g(f(x)), x) \circ(x, g(h(x))) \in$ $N \circ K$, where $K=K^{-1} \in \mathcal{E}_{X}$ is an entourage that shows that $g \circ h \sim_{\text {Sym }} i d_{X}$. Hence $(f(x), h(x)) \in(g \times g)^{-1}(N \circ K)$ and so $f \sim_{\text {Sym }} h$ since $(g \times g)^{-1}(N \circ K)=\left((g \times g)^{-1}(N \circ K)\right)^{-1} \in \mathcal{E}_{Y}$.

Note that, with an easy variation of the proof of Proposition 4.3. one can prove that every large-scale injective map $f:\left(X, \mathcal{E}_{X}\right) \rightarrow Y$ from a quasi-coarse space to a set has a partial Sym-coarse inverse, i.e., a map $g: Y^{\prime} \rightarrow\left(X, \mathcal{E}_{X}\right)$, where $Y^{\prime} \subseteq Y$, such that $g \circ f \sim_{\mathrm{Sym}} i d_{Y}$.

Theorem 4.4. Let $f:\left(X, \mathcal{E}_{X}\right) \rightarrow\left(Y, \mathcal{E}_{Y}\right)$ be a map between quasi-coarse spaces. Then the following are equivalent:

(a) $f$ is a Sym-coarse equivalence;

(b) $f:(X, \mathcal{E}) \rightarrow\left(Y, \mathcal{E}^{\prime}\right)$ is large-scale bijective, bornologous and weakly uniformly bounded copreserving;

(c) $f:(X, \mathcal{E}) \rightarrow\left(Y, \mathcal{E}^{\prime}\right)$ is large-scale bijective, bornologous and uniformly bounded copreserving;

(d) $f:(X, \mathcal{E}) \rightarrow\left(Y, \mathcal{E}^{\prime}\right)$ is large-scale surjective, bornologous and effectively proper;

(e) there exist two subspaces $X^{\prime} \subseteq X$ and $Y^{\prime} \subseteq Y$, which are large in $\left(X, \operatorname{Sym}\left(\mathcal{E}_{X}\right)\right)$ and in $\left(Y, \operatorname{Sym}\left(\mathcal{E}_{Y}\right)\right)$, respectively, and an asymorphism $f^{\prime}: X^{\prime} \rightarrow Y^{\prime}$.

Proof. The equivalences (b) $\leftrightarrow(\mathrm{c}) \leftrightarrow(\mathrm{d})$ follow from Proposition 1.6.

(a) $\rightarrow$ (d) Fix and entourage $E \in \mathcal{E}_{Y}$ and let $(x, y) \in(f \times f)^{-1}(E)$ be an arbitrary element in its preimage. Let $M=\{(x, g(f(x))),(g(f(x)), x) \mid x \in X\} \in \mathcal{E}_{X}$. Then

$$
(x, y)=(x, g(f(x))) \circ(g(f(x)), g(f(y))) \circ(g(f(y)), y) \in M \circ(g \times g)(E) \circ M \in \mathcal{E}_{X} .
$$

Hence, $f$ is effectively proper. Moreover, $f$ is large-scale surjective, since $\operatorname{Sym}\left(\mathcal{E}_{Y}\right) \subseteq \mathcal{E}_{Y}$ and, if $N=\{(y, f(g(y))) \mid y \in$ $Y$ \}, then the entourage $N \cup N^{\prime} \in \operatorname{Sym}\left(\mathcal{E}_{Y}\right) \subseteq \mathcal{E}_{Y}$ witnesses the property.

(d) $\rightarrow$ (e) Suppose that $f: X \rightarrow Y$ satisfies item (d). Let $X^{\prime} \subseteq X$ be a subset with the following property: for every $x \in X,\left|X^{\prime} \cap f^{-1}(f(x))\right|=1$. Then $f^{\prime}=\left.f\right|_{X^{\prime}}: X^{\prime} \rightarrow Y^{\prime}$, where $Y^{\prime}=f(X)=f\left(X^{\prime}\right)$, is bijective. Moreover, $f^{\prime}:\left(X^{\prime},\left.\mathcal{E}_{X}\right|_{X^{\prime}}\right) \rightarrow\left(Y^{\prime},\left.\mathcal{E}_{Y}\right|_{Y^{\prime}}\right)$ is bornologous and effectively proper, since it is a restriction of $f$. Finally, since $f: X \rightarrow Y$ has uniformly bounded fibers, $X^{\prime}$ is large in $\left(X, \operatorname{Sym}\left(\mathcal{E}_{X}\right)\right)$.

(e) $\rightarrow$ (a) Let $M=M^{-1} \in \mathcal{E}_{X}$ be an entourage such that $M\left[X^{\prime}\right]=X$. Then define a map $h: X \rightarrow X^{\prime}$ as follows: if $x \in X^{\prime}$, then $h(x)=x$, and, if otherwise $x \in X \backslash X^{\prime}$, then $h(x)$ is a point such that $(h(x), x) \in M$. Similarly we can define a map $k: Y \rightarrow Y^{\prime}$. We claim that $h$ and $k$ are bornologous. Let $E \in \mathcal{E}_{X}$. Then note that $(h \times h)(E) \subseteq M \circ E \circ E \in \mathcal{E}_{X}$ and thus $h$ is bornologous. The same property can be similarly proved for $k$. Then the maps $f=f^{\prime} \circ h$ and $g=\left(f^{\prime}\right)^{-1} \circ k$ are bornologous. We claim that $g$ is a Sym-coarse inverse of $f$. For every $x \in X$, since $\left.k\right|_{Y^{\prime}}=i d_{Y^{\prime}}$,

$$
(x, g(f(x)))=\left(x,\left(f^{\prime}\right)^{-1}\left(k\left(f^{\prime}(h(x))\right)\right)\right)=\left(x,\left(f^{\prime}\right)^{-1}\left(f^{\prime}(h(x))\right)\right)=(x, h(x)) \in M,
$$

and thus $g \circ f \sim_{\mathrm{Sym}} i d_{X}$. The other request can be similarly proved.

Proposition 4.5. Let $\left(X, \mathcal{E}_{X}\right)$ and $\left(Y, \mathcal{E}_{Y}\right)$ be two Sym-coarsely equivalent quasi-coarse spaces. If $\left(X, \mathcal{E}_{X}\right)$ is a coarse space, then so it is $\left(Y, \mathcal{E}_{Y}\right)$.

Proof. Let $f: X \rightarrow Y$ be a Sym-coarse equivalence and let $g: Y \rightarrow X$ be a Sym-coarse inverse of $f$. Moreover, let $E=E^{-1} \in \mathcal{E}_{X}$ and $F^{-1}=F \in \mathcal{E}_{Y}$ be two symmetric entourages which witness that $g \circ f \sim_{\text {Sym }} i d_{X}$ and $f \circ g \sim_{\text {Sym }} i d_{Y}$, respectively. Then, for every $K \in \mathcal{E}_{Y}$ and $(x, y) \in K$,

$$
(y, x)=(y, f(g(y))) \circ(f(g(y)), f(g(x))) \circ(f(g(x)), x) \in F \circ(f \times f)\left(((g \times g)(K))^{-1}\right) \circ F \in \mathcal{E}_{Y},
$$

and then $K^{-1} \in \mathcal{E}_{Y}$.

Let $\mathcal{X}$ be a category and $\sim$ be a congruence on $\mathcal{X}$, i.e., for every $X, Y \in \mathcal{X}, \sim$ is an equivalence relation in Mor $\mathcal{X}(X, Y)$ such that, for every $f, g \in \operatorname{Mor}_{\mathcal{X}}(X, Y)$ and $h, k \in \operatorname{Mor}_{\mathcal{X}}(Y, Z), h \circ f \sim k \circ g$, whenever $f \sim g$ and $h \sim k$. Hence the quotient category $\mathcal{X} / \sim$ can be defined as the one whose objects are the same of $\mathcal{X}$ and whose morphisms are equivalence classes of morphisms of $\mathcal{X}$, i.e., $\operatorname{Mor}_{\mathcal{X} / \sim}(X, Y)=\left\{[f]_{\sim} \mid f \in \operatorname{Mor}_{\mathcal{X}}(X, Y)\right\}$, for every $X, Y \in \mathcal{X} / \sim$. For example, the closeness relation $\sim$ is a congruence in Coarse and so the quotient category Coarse/ can be defined ([6]).

Let $\mathcal{X}$ be one of the categories Entou, SCoarse or QCoarse and let $\mathrm{F}: \mathcal{X} \rightarrow$ Coarse be a functor. As it is shown in the first part of this section, an equivalence relation $\sim_{\mathrm{F}}$ on $\mathcal{X}$ can be induced, which is actually a congruence (it easily follows, since $\mathrm{F}$ is a functor and $\sim$ is a congruence). Hence it is natural to produce the quotient category $\mathcal{X} / \sim_{\mathrm{F}}$ and to compare it with Coarse/ . In particular QCoarse/ $\sim_{\mathrm{Sym}}$ is worth being investigated. 


\subsection{Characterisation of some special classes of quasi-coarse spaces}

Proposition 4.6. Let $X$ and $Y$ be two Sym-coarsely equivalent quasi-coarse spaces. Then:

(a) $X$ is connected if and only if $Y$ is connected;

(b) $X$ is strongly connected if and only if $Y$ is strongly connected;

(c) $X$ is uniformly connected if and only if $Y$ is uniformly connected.

Proof. First of all, note that all those properties are invariant under asymorphism. Thanks to Theorem 4.4(e), it is enough to prove the claim when $X$ is a large subspace of $\operatorname{Sym}(Y)$, which can be easily shown.

Lemma 4.7. Let $\left(X, \mathcal{E}_{X}\right)$ and $\left(Y, \mathcal{E}_{Y}\right)$ be two Sym-coarsely equivalent quasi-coarse spaces. Then $\mathrm{cf} \mathcal{E}_{X}=\mathrm{cf} \mathcal{E}_{Y}$.

Proof. By applying Theorem 4.4, we can assume that $Y$ is an entourage subspace of $X$ and the inclusion map $i: Y \rightarrow X$ is large-scale surjective. It is trivial that $\operatorname{cf} \mathcal{E}_{Y} \leq \operatorname{cf} \mathcal{E}_{X}$. Let $f: X \rightarrow Y$ be a Sym-coarse inverse of $i$ and $M=M^{-1} \in \mathcal{E}_{X}$ be an entourage such that $(x, f(x)) \in M$, for every $x \in X$. Then, for every base $\left\{E_{i}\right\}_{i \in I}$ of $\mathcal{E}_{Y}$, we claim that $\left\{M \circ E_{i} \circ M\right\}_{i}$ is a base of $\mathcal{E}_{X}$, and thus $\operatorname{cf} \mathcal{E}_{X} \leq \operatorname{cf} \mathcal{E}_{Y}$. In fact, let $F \in \mathcal{E}_{X}$ and $i \in I$ be an index such that $\left.F\right|_{Y \times Y} \subseteq E_{i}$. Then $F \subseteq M \circ E_{i} \circ M$.

We are now ready to prove the generalisations of some classical classification results in the framework of quasi-coarse spaces (17, Theorems 9.1, 9.2], [20, Theorem 2.11]). The following results, together with Proposition 1.5 give a complete characterisation of metric entourage structures.

Theorem 4.8. Let $(X, \mathcal{E})$ be a quasi-coarse space. The following properties are equivalent:

(a) there exists an extended quasi-metric $d: X \times X \rightarrow[0, \infty]$ on $X$ such that $\mathcal{E}=\mathcal{E}_{d}$;

(b) there exists an extended quasi-metric space $(Y, d)$ which is Sym-coarsely equivalent to $(X, \mathcal{E})$;

(c) $\operatorname{cf} \mathcal{E} \leq \omega$.

Proof. The implications (a) $\rightarrow$ (b) $\rightarrow$ (c) are trivial: in particular, (b) $\rightarrow$ (c) is implied by Lemma 4.7 .

(c) $\rightarrow$ (a) Let $\left\{F_{n}\right\}_{n}$ be a base of $\mathcal{E}$ as in the proof of Proposition 1.5)(a) with the following further property: for every $m, n \in \mathbb{N}, F_{m} \circ F_{n} \subseteq F_{m+n}$. We claim that the map $d: X \times X \rightarrow[0, \infty]$ defined as in (3) is an extended quasi-metric and, in order to show it, proving that $d$ satisfies the triangle inequality is enough. Let $x, y, z \in X$ be three arbitrary points. The inequality $d(x, z) \leq d(x, y)+d(y, z)$ trivially holds if $d(x, y)=\infty$ or $d(y, z)=\infty$. Suppose now that $d(x, y) \leq m$ and $d(y, z) \leq n$. Then $(x, z)=(x, y) \circ(y, z) \in F_{m} \circ F_{n} \subseteq F_{m+n}$ and thus $d(x, z) \leq m+n$. Finally, the equality $\mathcal{E}=\mathcal{E}_{d}$ can be easily proved.

A quasi-coarse space satisfying the hypothesis of the previous theorem is called quasi-metrizable. Since the extended quasi-metric defined in the proof of Theorem 4.8 is a quasi-metric if and only if the quasi-coarse space is strongly connected, in view of Proposition 4.6. Theorem 4.8 can be specialised as follows.

Corollary 4.9. Let $(X, \mathcal{E})$ be a quasi-coarse space. The following properties are equivalent:

(a) there exists a quasi-metric $d$ on $X$ such that $\mathcal{E}=\mathcal{E}_{d}$;

(b) there exists a quasi-metric space $(Y, d)$ which is Sym-coarsely equivalent to $(X, \mathcal{E})$;

(c) $(X, \mathcal{E})$ is strongly connected and $\mathrm{cf} \mathcal{E} \leq \omega$.

[20. Proposition 2.1.1] implies that the extended quasi metrics in Theorem 4.8 and the quasi-metrics in Corollary 4.9 can be taken as extended metrics and metrics, respectively, if and only if the initial space is a coarse space.

Finally we can answer to a problem posed by Protasov and Banakh [17, Problem 9.4].

Theorem 4.10. Let $(X, \mathcal{E})$ be a connected quasi-coarse space. Then the following properties are equivalent:

(a) $(X, \mathcal{E})$ is a graphic quasi-coarse space;

(b) $(X, \mathcal{E})$ is Sym-coarsely equivalent to a graphic quasi-coarse space;

(c) $(X, \mathcal{E})$ is uniformly weakly connected and it is quasi-metrizable.

Proof. The implications (a) $\rightarrow$ (b) and (b) $\rightarrow$ (c) are trivial.

(c) $\rightarrow$ (a) Let $d$ be an extended quasi-metric such that $\mathcal{E}=\mathcal{E}_{d}$. Let $n$ be a natural number such that $\mathcal{E}_{d}$ is uniformly connected with parameter $E_{n}$. Finally, define a graph $\Gamma=(X, E)$, whose edges are the pairs $(x, y) \in X \times X$ such that $y \in E_{n}[x]$. Then the graphic quasi-ballean associated to the graph $\Gamma$ is asymorphic to $\mathcal{E}_{d}$ and so to $\mathcal{E}$.

In the previous theorem, the request that the quasi-coarse space is uniformly connected can be easily relaxed.

Corollary 4.11. Let $(X, \mathcal{E})$ be a quasi-coarse space. Then the following are equivalent:

(a) $(X, \mathcal{E})$ is a graphic quasi-coarse space;

(b) $(X, \mathcal{E})$ is Sym-coarsely equivalent to a graphic quasi-coarse space;

(c) $(X, \mathcal{E})$ is quasi-metrizable and there exists $E \in \mathcal{E}$ such that each connected component is uniformly connected with parameter $E$. 


\section{References}

[1] J. Adámek, H. Herrlich, G. E. Strecker, Abstract and Concrete Categories. The Joy of Cats, online edition, http://katmat.math.uni-bremen.de/acc/acc.pdf (2005).

[2] G. C. L. Brümmer, Topological categories, Topology Appl. 18, (1984) 27-41.

[3] E. Čech, Topological spaces, Academia, Prague, 1966.

[4] D. Dikranjan, I. Protasov, K. Protasova, N. Zava, Balleans, hyperballeans and ideals, Appl. Topology, to appear.

[5] D. Dikranjan, W. Tholen, Categorical Structure of Closure Operators with Applications to Topology, Algebra and Discrete Mathematics and its Applications, Kluwer Academic Publishers, Dordrecht, The Netherlands (1995).

[6] D. Dikranjan, N. Zava, Some categorical aspects of coarse spaces and balleans, Topology Appl., 225 (2017), 164-194.

[7] J. Dydak, C. S. Hoffland, An alternative definition of coarse structures, Topology Appl. 155 (2008), no. 9, 1013-1021.

[8] P. de la Harpe, Topics in geometric group theory. Chicago Lectures in Math., the University of Chicago Press, Chicago (2000).

[9] F. Hausdorff, Set theory, Second edition, translated from the German by John R. Aumann et al Chelsea Publishing Co., New York 1962352 pp. 04.00

[10] J. R. Isbell, Uniform Spaces, American Mathematical Society (1964).

[11] P. Fletcher, W. F. Lindgren, Quasi-Uniform Spaces, Lecture Notes Pure Appl. Math., 77, Dekker, New York (1982).

[12] H.-P. Kunzi, Nonsymmetric distances and teir associated topologies: about the origins of basic ideals in the area of asymmetric topology, in Handbook of the history of general topology, Vol. 3, 853-968, Hist. Topol., 3, Kluwer Acad. Publ., Dordrecht, 2001.

[13] P. W. Nowak, G. Yu, Large Scale Geometry. European Mathematical Society, 2012.

[14] A. Nicas, D. Rosenthal, Coarse structures on groups, Topology Appl. 159 (14) (2012) 3215-3228.

[15] J. Picado, A. Pultr, Cover quasi-uniformities in frames, Topology Appl. 158 (2011), 7, 869-881.

[16] I. Protasov, Asymptotic proximities, Applied Gen. Top., 9 (2008), no. 2, 189-195.

[17] I. Protasov, T. Banakh, Ball Structures and Colorings of Groups and Graphs, Mat. Stud. Monogr. Ser 11, VNTL, Lviv, 2003.

[18] I. V. Protasov, O. I. Protasova, Sketch of group balleans, Matematychni Studii, 22 (2004), no. 1, 10-20.

[19] I. Protasov, K. Protasova, On hyperballeans of bounded geometry, K. European Journal of Mathematics (2018), https://doi.org/10.1007/s40879-018-0236-y.

[20] I. Protasov, M. Zarichnyi, General Asymptology, 2007 VNTL Publishers, Lviv, Ukraine.

[21] J. Roe, Lectures on Coarse Geometry, Univ. Lecture Ser., vol. 31, American Mathematical Society, Providence RI, 2003.

[22] W. A. Wilson, On Quasi-Metric Spaces, Amer. J. Math. 53 (1931), no. 3, 675-684.

[23] N. Zava, Cowellpoweredness and closure operators in coarse categories, submitted. 